\title{
Crack identification in elastically restrained vibrating rods
}

\author{
José Fernández-Sáez \\ Department of Continuum Mechanics and Structural Analysis, University Carlos III of \\ Madrid, Avda. de la Universidad 30, 28911 Leganés, Madrid, Spain. E-mail: \\ ppfer@ing.uc3m.es \\ Antonino Morassi \\ Corresponding author. Polytechnic Department of Engineering and Architecture, University \\ of Udine, via Cotonificio 114, 33100 Udine, Italy. Tel.: +39 0432 558739; fax: +39 0432 \\ 558700. E-mail: antonino.morassi@uniud.it \\ Lourdes Rubio \\ Department of Mechanical Engineering, University Carlos III of Madrid, Avda. de la \\ Universidad 30, 28911 Leganés, Madrid, Spain. E-mail: Irubio@ing.uc3m.es
}

\begin{abstract}
In this paper we consider the problem of identifying an open crack in a longitudinally vibrating rod with smooth variable profile by minimal eigenfrequency data. The crack is assumed to be open during vibration and it is modelled by an elastic spring acting along the beam axis. Most, if not all, the results available in the literature for this inverse problem refer to ideal end conditions, that is the rod is either under free or supported end conditions. As an example of almost optimal result, it is known that the knowledge of the fundamental (positive) natural frequency of the rod under free-free and cantilever end conditions allows for the unique determination of the crack, without any restriction on the damage severity. In this paper we show that the analysis of the analogous crack identification problem for rods under elastically restrained end conditions leads to different results and that, in general, the knowledge of the fundamental frequency belonging to two spectra associated to different end conditions is not sufficient for the uniqueness of the solution. The method we used to solve the inverse problem is of constructive type and it is based on general properties of
\end{abstract}

Preprint submitted to International Journal of Non-Linear Mechanics December 17, 2016 
the eigenfrequencies as functions of the position and severity of the crack. The identification procedure has been tested numerically on rods under various damage scenarios. Numerical results agree well with the theory, even in presence of noisy input data.

Keywords: Crack identification, eigenfrequencies, rods, inverse problems

\section{Introduction}

The inverse problem of determining a crack in a beam from natural frequency measurements has attracted a lot of attention in the last thirty years, see, among other contributions, the results obtained in [1]-[21]. Inverse problems of this class are usually ill-posed according to Hadamard's definition, and one of the main issues is the uniqueness of the solution, that is the selection of a suitable set of natural frequency data which ensure the unique determination of the defect [22] (Chapter 15). Moreover, from the point of view of applications, it is very important to have at disposal a reconstruction algorithm for crack identification.

The prototype of these inverse problems is the determination of a single open crack in a straight thin elastic beam from measurement of natural frequencies of the longitudinal vibration. The crack can be modelled by means of a massless translational elastic spring located at the damaged cross-section, with stiffness depending on the geometry of the cross-section and on the mechanical properties of the material. This concentrated flexibility model of cracked rod proved to have accuracy comparable to that of the classical model of undamaged rod, in a large frequency range. We refer to [23] and [24] for a justification of the model via linear fracture mechanics and asymptotic analysis, respectively.

Assuming that the undamaged configuration of the defected rod is completely known, that is the rod profile, material properties and end conditions are given, the diagnostic problem consists in determining the position $s$ of the crack and the stiffness $K$ of the elastic spring simulating the crack. Since only two unknowns are involved, it is expected that at least two natural frequency 
data must be considered in order to formulate and solve the inverse problem. of view, the inverse problem when a minimal set of eigenfrequency data is known.

A first series of results along this line of research was obtained for uniform rods with a single small crack, namely, for $K$ large enough. Narkis [25] proved that the measurement of the first two (positive) natural frequencies of a free-

30 fee rod are enough to localize uniquely the crack, up to a symmetric position. Narkis's theory was extended in [26] by showing that the choice of the first two natural frequencies is optimal in order to avoid non-uniqueness. Later on, Dilena and Morassi [27] showed that the fundamental frequency of a uniform rod under free-free and supported-free (e.g., cantilever) end conditions allows 35 for the unique determination of the crack, so removing the spurious solution due to the symmetry of the problem.

The first result on crack identification in a longitudinally vibrating rod with a single not necessarily small crack is due to Rubio et al. [28], who proved that Dilena and Morassi's result continue to hold even for large cracks, provided that the rod profile is uniform. The proof was based on a careful study of the frequency equation written for the two natural frequencies used as data. The general case of identification of a single crack having any level of severity in a rod with smooth variable profile has been recently considered by the authors of the present paper in [29]. Among other things, it was shown in [29] that Dilena 45 and Morassi's result [27] can be extended to this more general setting.

All the above results, and even the experimental research available in the literature ([1], [13], [30]-[35]), hold under the assumption that the boundary conditions at the ends of the rod are ideal, that is either the displacement vanishes (supported end) or the axial force vanishes (free end). Ideal end conditions can be considered as a simplified model of real end conditions. Therefore, it is of practical interest to study the crack detection problem in a longitudinally vibrating rod under elastically restrained (ER) end conditions, in which one or both the ends of the rod are attached to a fixed support by means of an elastic spring having assigned stiffness. This is the main goal of the present research. 
In this paper, we first show that a result analogous to that obtained in [29] does not hold for the present problem. More precisely, the knowledge of the fundamental frequency of a rod under ER-ER and ER-free end conditions does not always ensure the uniqueness of identification of the damage. As a positive result, instead, we prove that the measurement of the fundamental frequency of

${ }_{60}$ the ER-ER rod and the first (positive) frequency of the free-free rod is sufficient for the unique identification of the damage, up to a symmetric position of the crack in case of symmetric rod. The obtained results show that end conditions play an important role in crack identification and, in addition, that the pair of eigenfrequency data must be properly selected in order to have unique solution of the inverse problem. Our analysis is based on a suitable extension of the socalled $\lambda$-Curves Method, that is the reconstruction procedure proposed in [29] to deal with the crack identification problem in rods having variable profile. We recall that the $\lambda$-Curves Method is mainly based on the analysis of the behavior of the eigenfrequencies as functions of the position and the severity of the crack.

The plane of the paper is as follows. Section 2 is devoted to the formulation of the inverse problem. Main results and the constructive method of identification are shown in Section 3. Results of numerical simulations are presented in Section 4. Proofs of some technical properties are collected, for completeness and for reader convenience, in Section 5 .

\section{2. Formulation of the diagnostic problem and main results}

The free undamped longitudinal vibrations with radian frequency $\omega$ of a straight thin rod of length $L$ and with a crack at the cross-section of abscissa $z_{d}, 0<z_{d}<L$, are governed by the following eigenvalue problem 


$$
\left\{\begin{array}{l}
\frac{d}{d z}\left(E \widehat{A}(z) \frac{d \widehat{u}(z)}{d z}\right)+\omega^{2} \gamma \widehat{A}(z) \widehat{u}(z)=0, \quad z \in\left(0, z_{d}\right) \cup\left(z_{d}, L\right), \\
E \widehat{A}(0) \frac{d \widehat{u}(z)}{d z}(0)=\widehat{h} \widehat{u}(0), \\
{\left[\left[E \widehat{A}\left(z_{d}\right) \frac{d \widehat{u}(z)}{d z}\left(z_{d}\right)\right]\right]=0,} \\
\widehat{K}\left[\left[\widehat{u}\left(z_{d}\right)\right]\right]=E \widehat{A}\left(z_{d}\right) \frac{d \widehat{u}(z)}{d z}\left(z_{d}\right), \\
E \widehat{A}(L) \frac{d \widehat{u}(z)}{d z}(L)=-\widehat{h} \widehat{u}(L),
\end{array}\right.
$$

where $\widehat{u}=\widehat{u}(z)$ is the spatial amplitude of the vibration and $\left[\left[\widehat{u}\left(z_{d}\right)\right]\right]=\lim _{z \rightarrow z_{d}^{+}} \widehat{u}(z)-$ $\lim _{z \rightarrow z_{d}^{-}} \widehat{u}(z)$. In (1)-(5), $\widehat{A}=\widehat{A}(z)$ denotes the area of the transversal crosssection of the rod. The function $\widehat{A}$ is assumed to be strictly positive and continuously differentiable in $[0, L]$. The (constant) Young's modulus of the material is denoted by $E, E>0 ; \gamma$ is the (constant) volume mass density, $\gamma>0$. The crack is assumed to remain open during vibration and it is modelled as a longitudinally elastic spring with stiffness $\widehat{K}$, see, for example, [23] and [24]. The value of $\widehat{K}$ depends on the geometry of the beam and on the material, see Section 4 for an application to rectangular cross-section and transversal crack. Both the ends of the rod are restrained to fixed supports by means of a linearly elastic spring of stiffness $\widehat{h}, 0<\widehat{h}<\infty$. Even if part of the following results hold under more general hypotheses, we shall consider rods whose cross-sectional area profile is a symmetric function with respect to the mid point of the rods axis, e.g.

$$
\widehat{A}=\widehat{A}(L-z), \quad z \in[0, L] .
$$

By setting

$$
x=\frac{z}{L}, \quad u(x)=\widehat{u}(z), \quad A(x)=\widehat{A}(z), \quad a(x)=\frac{A(x)}{A\left(x_{0}\right)}, \quad \text { for a given } x_{0} \in[0, L],
$$

so the eigenvalue problem (1)-(5) can be written in dimensionless form as

$$
\left\{\begin{array}{l}
\left(a u^{\prime}\right)^{\prime}+\lambda a u=0, \quad x \in(0, s) \cup(s, 1) \\
a(0) u^{\prime}(0)=h u(0) \\
{\left[\left[a u^{\prime}(s)\right]\right]=0} \\
K[[u(s)]]=a(s) u^{\prime}(s) \\
a(1) u^{\prime}(1)=-h u(1)
\end{array}\right.
$$


where

$s=\frac{z_{d}}{L} \in(0,1), \quad K=\frac{\widehat{K} L}{E A\left(x_{0}\right)} \in(0, \infty), \quad \lambda=\frac{\gamma L^{2} \omega^{2}}{E}, \quad h=\frac{\widehat{h} L}{E A\left(x_{0}\right)} \in(0, \infty)$,

and

$$
a(x)=a(1-x) \quad \text { in }[0,1], \quad a \in C^{1}([0,1]), \quad a(x) \geq a_{0}>0 \quad \text { in }[0,1],
$$

where $a_{0}$ is a given constant; see Figure 1 . Hereinafter, we use the notation $(\cdot)^{\prime}=\frac{d(\cdot)}{d x}$ to indicate $x$-differentiation. Under the above assumptions, it is known (see, for example, [36]) that there exists a numerable sequence of real, positive, simple eigenvalues $\left\{\lambda_{n}\right\}_{n=1}^{\infty}$ of (8)-(12) such that

$$
0<\lambda_{1}<\lambda_{2}<\ldots, \quad \lim _{n \rightarrow \infty} \lambda_{n}=+\infty
$$

In order to formulate our first result, we introduce the auxiliary eigenvalue problem obtained by removing the elastic constraint at $x=1$ in (8)-(12), namely (see Figure 2)

$$
\left\{\begin{array}{l}
\left(a v^{\prime}\right)^{\prime}+\mu a v=0, \quad x \in(0, s) \cup(s, 1), \\
a(0) v^{\prime}(0)=h v(0), \\
{\left[\left[a v^{\prime}(s)\right]\right]=0,} \\
K[[v(s)]]=a(s) v^{\prime}(s), \\
a(1) v^{\prime}(1)=0,
\end{array}\right.
$$

where $a, h, K$ are defined as above,

$$
\mu=\frac{\gamma L^{2} \omega^{2}}{E}
$$

and

$$
0<\mu_{1}<\mu_{2}<\ldots, \quad \lim _{n \rightarrow \infty} \mu_{n}=+\infty .
$$

It is known (see, for example, [37]) that the eigenvalues of (8)-(12) and (16)-(20) interlace, that is

$$
0<\mu_{1}<\lambda_{1}<\mu_{2}<\lambda_{2}<\ldots
$$

The crack identification problem in a longitudinally vibrating rod under elastically restrained end conditions is considered in the following theorem. 
Theorem 2.1. There exists a constructive procedure for the determination of the position and severity of the crack in terms of the measurement of the fundamental eigenvalue $\lambda_{1}$ and $\mu_{1}$ of the problems (8)-(12) and (16)-(20), respectively. The determination of the crack is not necessarily unique. The reconstruction method provides all the solutions of the inverse problem.

If the elastic constraint at $x=0$ in (17) is removed, then the rod is under freefree end conditions and the longitudinal vibration is governed by the eigenvalue problem

$$
\left\{\begin{array}{l}
\left(a t^{\prime}\right)^{\prime}+\nu a t=0, \quad x \in(0, s) \cup(s, 1), \\
a(0) t^{\prime}(0)=0, \\
{\left[\left[a t^{\prime}(s)\right]\right]=0,} \\
K[[t(s)]]=a(s) t^{\prime}(s), \\
a(1) t^{\prime}(1)=0,
\end{array}\right.
$$

where $\nu, a, K$ are defined as above and (see, for example, [36])

$$
0=\nu_{0}<\nu_{1}<\nu_{2}<\ldots, \quad \lim _{n \rightarrow \infty} \nu_{n}=+\infty .
$$

Our second identification result is as follows.

Theorem 2.2. The measurement of the fundamental eigenvalue $\lambda_{1}$ of the problem (8)-(12) and the first positive eigenvalue $\nu_{1}$ of the problem (24)-(28) allows for the unique determination of the crack, up to a symmetric position. The identification procedure is constructive.

We conclude this section by highlighting a peculiarity of our approach, namely the need to resort to information belonging to two spectra associated to different end conditions. More generally, one of the most celebrated results of the inverse spectral theory for Sturm-Liouville differential operators shows that the unique determination of the rod profile $a=a(x)$ (in absence of crack) requires the knowledge of two full spectra associated with two sets of boundary conditions [38]. In the inverse problem studied in this paper, the unknowns are 
reduced to two, the position and intensity of the damage, and in view of the above general result the use of information on eigenvalues belonging to different spectra is rather expected. In addition, the damage identification results can be considered almost optimal, since the number of unknowns equals the number of resonant frequency data. Finally, it is important to recall that the eigenvalues needed to formulate and solve the diagnostic problem are the lower order ones of both spectra, which are known to be easier to measure experimentally and for which the analytical model of the axially vibrating beam is very accurate.

The next section is devoted to the proof of Theorems 2.1 and 2.2 .

\section{Proof of the main results via the $\lambda$-Curves Method}

\subsection{Proof of Theorem 2.1}

The identification method we shall use is strongly based on the analysis of the behavior of the fundamental eigenvalues $\lambda_{1}$ and $\mu_{1}$ of (8)-(12) and (16)(20), respectively, as functions of the damage parameters $s$ and $K$. It can be shown that the analysis is simplified by introducing an equivalent eigenvalue problem to both (8)-(12) and (16)-(20).

Proposition 3.1. Let $(\lambda, u)$ be an eigenpair of (8)-(12). Then $(\lambda, w), \lambda>0$, is an eigenpair of the problem (see Figure 3)

$$
\left\{\begin{array}{l}
\left(b w^{\prime}\right)^{\prime}+\lambda b w=0, \quad x \in(0, s) \cup(s, 1), \\
b(0) w^{\prime}(0)=-\lambda \mathcal{M} w(0) \\
{[[w(s)]]=0} \\
{\left[\left[b w^{\prime}(s)\right]\right]=-\lambda m w(s)} \\
b(1) w^{\prime}(1)=\lambda \mathcal{M} w(1)
\end{array}\right.
$$

where

$$
w=a u^{\prime} \quad \text { in }(0, s) \cup(s, 1), \quad b=a^{-1} \quad \text { in }[0,1], \quad m=K^{-1}, \quad \mathcal{M}=h^{-1} .
$$

Conversely, if $(\lambda, w)$ is an eigenpair of (30)-(34) with $\lambda>0$, then $(\lambda, u)$ is an eigenpair of (8)-(12), where $u$ is such that

$$
u=b w^{\prime} \quad \text { in }(0, s) \cup(s, 1), \quad a=b^{-1} \quad \text { in }[0,1], \quad K=m^{-1}, \quad h=\mathcal{M}^{-1} \text {. }
$$


Proof. The proof of the proposition follows the same lines of the proof of the analogous result for $h=0$ contained in [29] (Proposition 2.1).

It should be noticed that problem (30)-(34) describes the free longitudinal vibration of a rod with cross-sectional area $b=b(x)$, carrying a point mass $m$ at $x=s$, and with two (equal) point masses $\mathcal{M}$ at the ends $x=0$ and $x=1$. It should be also noticed that the smallest eigenvalue of (30)-(34) vanishes, e.g., $\lambda_{0}=0$, since it corresponds to the rigid translation of the rod $w_{0}=$ const. in $[0,1]$.

By arguments analogous to those adopted for Proposition 3.1, one can prove the following result.

Proposition 3.2. Let $(\mu, v)$ be an eigenpair of (16)-(20). Then $(\mu, y), \mu>0$, is an eigenpair of the problem (see Figure 4)

$$
\left\{\begin{array}{l}
\left(b y^{\prime}\right)^{\prime}+\mu b y=0, \quad x \in(0, s) \cup(s, 1), \\
b(0) y^{\prime}(0)=-\mu \mathcal{M} y(0), \\
{[[y(s)]]=0,} \\
{\left[\left[b y^{\prime}(s)\right]\right]=-\lambda m y(s),} \\
y(1)=0,
\end{array}\right.
$$

where

$$
y=a v^{\prime} \quad \text { in }(0, s) \cup(s, 1), \quad b=a^{-1} \quad \text { in }[0,1], \quad m=K^{-1}, \quad \mathcal{M}=h^{-1} .
$$

Conversely, if $(\mu, y)$ is an eigenpair of $(37)-(41)$, then $(\mu, v)$ is an eigenpair of (16)-(20), with

$$
v=b y^{\prime} \quad \text { in }(0, s) \cup(s, 1), \quad a=b^{-1} \quad \text { in }[0,1], \quad K=m^{-1}, \quad h=\mathcal{M}^{-1} .
$$

The eigenvalue problem (37)-(41) describes the free longitudinal vibration of a rod carrying a point mass $m$ at $x=s$ and a point mass $\mathcal{M}$ at $x=0$, with the right end supported (cantilever).

Basing on the equivalence stated in Propositions 3.1 and 3.2, the inverse problem of determining a crack $(s, K)$ in a rod can be reformulated as the 
inverse problem of determining the intensity $m$ and the position $s$ of a point mass from a suitable pair of natural frequency data. This equivalent formulation of the inverse problem will be adopted in the sequel.

In what follows we shall often compare the eigenpairs of the problem (30)(34) for finite no-vanishing $m$ and for $s \in(0,1)$ to those obtained by taking $m=0$. We shall denote by $\left\{\lambda_{n}^{U}, w_{n}^{U}\right\}, n \geq 0$, the $n$th eigenpair of the unperturbed problem

$$
\left\{\begin{array}{l}
\left(b w^{U^{\prime}}\right)^{\prime}+\lambda^{U} b w^{U}=0, \quad x \in(0,1) \\
b(0) w^{U^{\prime}}(0)=-\lambda^{U} \mathcal{M} w^{U}(0) \\
b(1) w^{U^{\prime}}(1)=\lambda^{U} \mathcal{M} w^{U}(1)
\end{array}\right.
$$

where

$$
0=\lambda_{0}^{U}<\lambda_{1}^{U}<\lambda_{2}^{U}<\ldots
$$

Similarly, the unperturbed problem associated to (37)-(41) is

$$
\left\{\begin{array}{l}
\left(b y^{U^{\prime}}\right)^{\prime}+\mu^{U} b y^{U}=0, \quad x \in(0,1) \\
b(0) y^{U^{\prime}}(0)=-\mu^{U} \mathcal{M} y^{U}(0) \\
y^{U}(1)=0
\end{array}\right.
$$

where the eigenpairs $\left\{\mu_{n}^{U}, y_{n}^{U}\right\}, n \geq 1$, are such that

$$
0<\mu_{1}^{U}<\mu_{2}^{U}<\ldots
$$

Note that problems (44)-(46) and (48)-(50) can be formally obtained by taking $m=0$ in problem (30)-(34) and (37)-(41), respectively. By monotonicity results (see, for example, [37]), we have

$$
\begin{aligned}
& \lambda_{n-1}^{U} \leq \lambda_{n} \leq \lambda_{n}^{U}, \quad \text { for every } n \geq 1, \\
& \mu_{n-1}^{U} \leq \mu_{n} \leq \mu_{n}^{U}, \quad \text { for every } n \geq 1,
\end{aligned}
$$

where $\mu_{0}^{U}=0$.

The proof of Theorem 2.1 strongly relies on the behavior of the eigenvalues $\lambda_{1}$ and $\mu_{1}$ of (30)-(34) and (37)-(41) with respect to the parameters $m$ and $s$. 
The dependence of the eigenvalues on the parameter $m$, for a given position of the point mass $s$, is investigated in the next proposition.

Proposition 3.3. Let $\left(\lambda_{1}, w_{1}\right)$ and $\left(\lambda_{1}^{U}, w_{1}^{U}\right)$ be the first eigenpair of (30)-(34) and (44)-(46), respectively. We have:

i) $\lambda_{1}\left(\frac{1}{2}, m\right)=\lambda_{1}^{U}$ for every finite positive $m$.

ii) The function $\lambda_{1}=\lambda_{1}(s, m), s \in[0,1] \backslash\left\{\frac{1}{2}\right\}$, is a monotonically decreasing 165 function of $m$ in $[0, \infty)$.

iii) If $\lambda_{1}\left(s_{0}, m_{0}\right)=\lambda_{1}^{U}$ for some $s_{0} \in(0,1)$ and some $m_{0} \in(0, \infty)$, then $s_{0}=\frac{1}{2}$.

iv) If $w_{1}\left(s_{0}, s_{0}, m_{0}\right)=0$ for some $s_{0} \in(0,1)$ and some $m_{0} \in(0, \infty)$, then $s_{0}=\frac{1}{2}$.

Let $\left(\mu_{1}, y_{1}\right)$ and $\left(\mu_{1}^{U}, y_{1}^{U}\right)$ be the first eigenpair of (37)-(41) and (48)-(50), respectively. We have:

i) $\mu_{1}(1, m)=\mu_{1}^{U}$ for every finite positive $m$.

ii) The function $\mu_{1}=\mu_{1}(s, m), s \in[0,1)$, is a monotonically decreasing function of $m$ in $[0, \infty)$.

iii) If $\mu_{1}\left(s_{0}, m_{0}\right)=\mu_{1}^{U}$ for some $s_{0} \in[0,1]$ and some $m_{0} \in(0, \infty)$, then $s_{0}=1$.

The next proposition deals with the $\lambda$-s curves of the eigenvalues $\lambda_{1}$ and $\mu_{1}$ as functions of the position $s$ of the point mass, for a given intensity $m$.

Proposition 3.4. Let $\lambda_{1}, \mu_{1}$ be the first eigenpair of the problem (30)-(34), (37)-(41), respectively. Let $m$ be given , $0<m<\infty$. Then:

i) $\lambda_{1}=\lambda_{1}(s)$ is a continuously differentiable function in $[0,1]$, strictly increasing in $\left(0, \frac{1}{2}\right)$, with $\lambda_{1}(s)=\lambda_{1}(1-s)$ in $[0,1]$ and such that $0<\lambda_{1}(s) \leq \lambda_{1}^{U}$ in $[0,1], \lambda_{1}(0)<\lambda_{1}^{U}, \lambda_{1}\left(\frac{1}{2}\right)=\lambda_{1}^{U}, \frac{d \lambda_{1}}{d s}(0)>0, \frac{d \lambda_{1}}{d s}\left(\frac{1}{2}\right)=0$.

ii) $\mu_{1}=\mu_{1}(s)$ is a continuously differentiable, strictly increasing function in ${ }_{185}[0,1]$, such that $0<\mu_{1}(s) \leq \mu_{1}^{U}$ in $[0,1], \mu_{1}(0)<\mu_{1}^{U}, \mu_{1}(1)=\mu_{1}^{U}, \frac{d \mu_{1}}{d s}(0)>0$, $\frac{d \mu_{1}}{d s}(1)=0$. 
A proof of Proposition 3.3 and Proposition 3.4 will be presented in Section 5.

We are now in position to prove Theorem 2.1. The proof is constructive and leads to an algorithm for the determination of the unknown parameters $s$, $m$ from the knowledge of $\lambda_{1}$ and $\mu_{1}$. The proof follows the ideas presented in [29] to deal with the analogous problem for a cracked rod under free-free and supported-free end conditions (see Section 7 of the above mentioned paper). However, the present problem has some peculiarities which require a suitable modification of the identification procedure.

Let $\left\{\bar{\lambda}_{1}, \bar{\mu}_{1}\right\}$ be the experimental values of the first eigenvalue of (30)-(34) and (37)-(41), respectively, which are assumed to satisfy the inequalities

$$
0<\bar{\lambda}_{1} \leq \lambda_{1}^{U}, \quad 0<\bar{\mu}_{1}<\mu_{1}^{U} .
$$

Note that the upper bound of $\bar{\mu}_{1}$ is strict, since the first eigenvalue of (37)-(41) is always sensitive to the presence of the point mass $m$ for $s \in(0,1)$.

If $\bar{\lambda}_{1}=\lambda_{1}^{U}$, then, by symmetry, $s=\frac{1}{2}$. By Proposition $3.3, \mu_{1}=\mu_{1}\left(\frac{1}{2}, m\right)$ is a monotonically decreasing function of $m$, with $\lim _{m \rightarrow \infty} \mu_{1}\left(\frac{1}{2}, m\right)=0^{+}$.

Therefore, we can determine uniquely $m$ by solving the equation $\bar{\mu}_{1}=\mu\left(\frac{1}{2}, m\right)$.

In the sequel we shall consider the non-trivial condition

$$
0<\bar{\lambda}_{1}<\lambda_{1}^{U}
$$

Let us define $m_{\lambda}^{-}, m_{\mu}^{-} \in(0, \infty)$ such that

$$
\lambda_{1}\left(0, m_{\lambda}^{-}\right)=\bar{\lambda}_{1}, \quad \mu_{1}\left(0, m_{\mu}^{-}\right)=\bar{\mu}_{1} .
$$

Clearly, $m_{\lambda}^{-} \neq m_{\mu}^{-}($since $s \in(0,1))$ and

$$
m_{\lambda}^{-}<m, \quad m_{\mu}^{-}<m .
$$

We shall distinguish two main cases.

First Case. Let us assume

$$
\max \left\{m_{\lambda}^{-}, m_{\mu}^{-}\right\}=m_{\mu}^{-} .
$$


We construct the curve $\lambda_{1}=\lambda_{1}\left(s, m_{\mu}^{-}\right)$(see the dashed curve in Figure 5). This curve intersects the straight line $\lambda_{1}=\bar{\lambda}_{1}$ at two points, say, $P_{1 l}\left(m_{\mu}^{-}\right)$at the left of $s=\frac{1}{2}$, and $P_{1 r}\left(m_{\mu}^{-}\right)$at the right of $s=\frac{1}{2}$, with $s\left(P_{1 l}\left(m_{\mu}^{-}\right)\right) \in\left(0, \frac{1}{2}\right)$, ${ }_{205} s\left(P_{1 r}\left(m_{\mu}^{-}\right)\right)=1-s\left(P_{1 l}\left(m_{\mu}^{-}\right)\right)$.

Now, let us increase continuously the parameter $m$ from $m_{\mu}^{-}$. We obtain the curves $\lambda_{1}=\lambda_{1}(s, m)$ and $\mu_{1}=\mu_{1}(s, m)$ (see dotted curves in Figure 5) having intersection points $\left\{P_{1 l}(m), P_{1 r}(m)\right\}$ and $Q_{1}(m)$ with the straight lines $\lambda_{1}=\bar{\lambda}_{1}$ and $\mu_{1}=\bar{\mu}_{1}$, respectively. We have $s\left(P_{1 l}(m)\right) \in\left(0, \frac{1}{2}\right), s\left(P_{1 r}(m)\right)=$ $1-s\left(P_{1 l}(m)\right)$ for every $m \in\left(m_{\lambda}^{-}, \infty\right)$, and the point $P_{1 l}$ moves to the right whereas $P_{1 r}$ moves to the left as $m$ increases. The point $Q_{1}(m)$ is such that $s\left(Q_{1}(m)\right) \in(0,1)$ for every $m \in\left(m_{\mu}^{-}, \infty\right)$, it moves to the right as $m$ increases and $\lim _{m \rightarrow \infty} s\left(Q_{1}(m)\right)=0^{+}, s\left(Q_{1}\left(m_{\mu}^{-}\right)\right)=0$. It follows that, when $m$ is large enough (say, $m>m_{\frac{1}{2}}$, where $\mu_{1}\left(\frac{1}{2}, m_{\frac{1}{2}}\right)=\bar{\mu}_{1}$ ), the points $Q_{1}(m)$ and $P_{1 r}(m)$ move one toward each other, and there exists a unique value $\widetilde{m}$ such that $\widetilde{s}=s\left(Q_{1}(\widetilde{m})\right)=s\left(P_{1 r}(\widetilde{m})\right)$. The value $\widetilde{m}$ is the intensity of the mass and $\widetilde{s}$ is its positions. Conversely, when $m \in\left(m_{\mu}^{-}, m_{\frac{1}{2}}\right)$, by recalling the $s\left(Q_{1}\left(m_{\mu}^{-}\right)\right)=0$ and $s\left(P_{1 l}\left(m_{\mu}^{-}\right)\right) \in\left(0, \frac{1}{2}\right)$, there exists (at least) another solution $(\widehat{s}, \widehat{m})$ of the inverse problem, namely there exists $\widehat{m} \in\left(m_{\mu}^{-}, m_{\frac{1}{2}}\right)$ such that $\widehat{s}=s\left(Q_{1}(\widehat{m})\right)=$ ${ }_{220} s\left(P_{1 l}(\widehat{m})\right)$.

In conclusion, we have shown that in the First Case (58), the inverse problem has at least two solutions, namely, there are pairs $\left(m^{\prime}, s^{\prime}\right)$, with $m^{\prime} \in\left(m_{\mu}^{-}, m_{\frac{1}{2}}\right)$ and $s^{\prime} \in\left(\frac{1}{2}, 1\right)$, and $\left(m^{\prime \prime}, s^{\prime \prime}\right)$, with $m^{\prime \prime} \in\left(m_{\mu}^{-}, m_{\frac{1}{2}}\right)$ and $s^{\prime \prime} \in\left(0, \frac{1}{2}\right)$, which correspond to the same spectral data $\lambda_{1}$ and $\mu_{1}$. This situation does not occur under ideal end conditions (e.g., free-free and supported-free end conditions, see Section 7 in [29]).

Second Case. Let us assume

$$
\max \left\{m_{\lambda}^{-}, m_{\mu}^{-}\right\}=m_{\lambda}^{-} .
$$

We determine the curve $\mu_{1}=\mu_{1}\left(s, m_{\lambda}^{-}\right)$and we denote by $Q_{1}\left(m_{\lambda}^{-}\right)$its unique intersection point with the straight line $\mu_{1}=\bar{\mu}_{1}$. We distinguish two subcases, depending on the abscissa of $Q_{1}\left(m_{\lambda}^{-}\right)$with respect to $s=\frac{1}{2}$. 
Second Case - subcase a. Suppose that

$$
s\left(Q_{1}\left(m_{\lambda}^{-}\right)\right)<\frac{1}{2}
$$

230 parameter $m$ from $m_{\lambda}^{-}$. The curve $\lambda_{1}=\lambda_{1}(s, m)$ has two intersection points with the straight line $\lambda_{1}=\bar{\lambda}_{1}$, say $P_{1 l}(m)$ and $P_{1 r}(m)$, with $s\left(P_{1 l}(m)\right) \in\left(0, \frac{1}{2}\right)$ and $s\left(P_{1 r}(m)\right)=1-s\left(P_{1 l}(m)\right) \in\left(\frac{1}{2}, 1\right)$ for every $m>m_{\lambda}^{-}$. The point $P_{1 l}(m)$ moves to the right, whereas $P_{1 r}(m)$ moves to the left as $m$ increases, with ${ }_{235} s\left(P_{1 l}\left(m_{\lambda}^{-}\right)\right)=0, s\left(P_{1 r}\left(m_{\lambda}^{-}\right)\right)=1$. The point $Q_{1}(m)$ moves to the right and $\lim _{m \rightarrow \infty} s\left(Q_{1}(m)\right)=1$. Therefore, the points $Q_{1}$ and $P_{1 r}$ move one toward each other, and there exists a unique value $m^{*} \in\left(m_{\lambda}^{-}, m_{\frac{1}{2}}\right)$ (where $\left.s\left(Q_{1}\left(m_{\frac{1}{2}}\right)\right)=\frac{1}{2}\right)$ such that $s^{*}=s\left(Q_{1}\left(m^{*}\right)\right)=s\left(P_{1 r}\left(m^{*}\right)\right), s^{*} \in\left(\frac{1}{2}, 1\right)$, and the inverse problem has solution $\left(s^{*}, m^{*}\right)$.

It remains to check whether there exist other solutions $(m, s)$ with $s \in\left(0, \frac{1}{2}\right)$ and $m>m_{\lambda}^{-}$. If $s\left(P_{1 l}\left(m_{\frac{1}{2}}\right)\right)<s\left(Q_{1}\left(m_{\lambda}^{-}\right)\right)$, then there is no further solution. In fact, by increasing $m$ from $m_{\frac{1}{2}}$, the point $Q_{1}$ is such that $s\left(Q_{1}(m)\right)>\frac{1}{2}$, whereas $s\left(P_{1 l}(m)\right)<\frac{1}{2}$. Conversely, if the above condition is not satisfied, then (at least) another solution $(m, s)$ may exist with $s \in\left(0, \frac{1}{2}\right)$. In this last case, the inverse problem has more than one solution, as in the First Case.

Second Case - subcase B. Suppose that

$$
s\left(Q_{1}\left(m_{\lambda}^{-}\right)\right)>\frac{1}{2},
$$

see Figure 7. By increasing continuously $m$ from $m_{\lambda}^{-}$and arguing as in previous step, we can conclude that there exists a unique $m^{*} \in\left(m_{\lambda}^{-}, \infty\right)$ such that $s^{*}=$ $s\left(P_{1 r}\left(m^{*}\right)\right)=s\left(Q_{1}\left(m^{*}\right)\right)$, and the unique solution to our diagnostic problem is $\left.s^{*}, m^{*}\right)$.

Table 1 summarizes our findings.

\subsection{Proof of Theorem 2.2}

In order to prove Theorem 2.2, we first recall the auxiliary eigenvalue problem equivalent to (24)-(28) (see also [29], Proposition 2.1): 


$$
\left\{\begin{array}{l}
\left(b \zeta^{\prime}\right)^{\prime}+\nu b \zeta=0, \quad x \in(0, s) \cup(s, 1), \\
\zeta(0)=0, \\
{[[\zeta(s)]]=0,} \\
{\left[\left[b \zeta^{\prime}(s)\right]\right]=-\nu m \zeta(s),} \\
\zeta(1)=0,
\end{array}\right.
$$

where the usual notation has been used, namely $\zeta=a t^{\prime}, b=a^{-1}, m=K^{-1}$. Note that the eigenvalues of (62)-(66) are

$$
0<\nu_{1}<\nu_{2}<\ldots
$$

255 the analogous of Proposition 3.4 (see Theorem 5.5 in [29] for a proof).

Proposition 3.5. Let $\nu_{1}$ be the first eigenvalue of (62)-(66) and let $m$ be given, $m \in(0, \infty)$. Then $\nu_{1}=\nu_{1}(s)$ is a strictly decreasing function in $\left(0, \frac{1}{2}\right)$, with $\nu_{1}(s)=\nu_{1}(1-s)$ in $[0,1]$, and

$$
\nu_{1}\left(\frac{1}{2}\right)<\nu_{1}^{U}, \quad \nu_{1}(0)=\nu_{1}(1)=\nu_{1}^{U}, \quad \frac{d \nu_{1}}{d s}(0)=\frac{d \nu_{1}}{d s}\left(\frac{1}{2}\right)=\frac{d \nu_{1}}{d s}(1)=0 .
$$

Here, $\nu_{1}^{U}$ denotes the first eigenvalue of the unperturbed problem obtained by taking $m=0$ in $(62)-(66)$.

Now we prove Theorem 2.2.

Let us denote by $\left\{\bar{\lambda}_{1}, \bar{\nu}_{1}\right\}$ the experimental (given) values of the 1 st eigenvalue of (30)-(34) and (62)-(66), respectively, which are assumed to satisfy the inequalities

$$
0<\bar{\lambda}_{1}<\lambda_{1}^{U}, \quad 0<\bar{\nu}_{1}<\nu_{1}^{U}
$$

Note that if $\bar{\lambda}_{1}=\lambda_{1}^{U}$, then $s=\frac{1}{2}$, and the value of $m$ can be uniquely determined by solving the equation $\bar{\nu}_{1}=\nu_{1}\left(\frac{1}{2}, m\right)$.

By symmetry, let us assume $s \in\left(0, \frac{1}{2}\right)$. Let us define $m_{\lambda}^{-}, m_{\nu}^{-} \in(0, \infty)$ such that

$$
\lambda_{1}\left(0, m_{\lambda}^{-}\right)=\bar{\lambda}_{1}, \quad \nu_{1}\left(\frac{1}{2}, m_{\nu}^{-}\right)=\bar{\nu}_{1}
$$


We have

$$
m_{\lambda}^{-}<m, \quad m_{\nu}^{-}<m
$$

and we shall study two main cases.

First CASE.

$$
\max \left\{m_{\lambda}^{-}, m_{\nu}^{-}\right\}=m_{\nu}^{-},
$$

see Figure 8. We construct the curve $\lambda_{1}=\lambda_{1}\left(s, m_{\nu}^{-}\right)$(dashed curve in Figure 8). The curve $\lambda_{1}=\lambda_{1}\left(s, m_{\nu}^{-}\right)$intersects the straight line $\lambda_{1}=\bar{\lambda}_{1}$ at the point $P_{1}$. Let us increase continuously the parameter $m$ from $m_{\nu}^{-}$. We obtain the curves $\lambda_{1}=\lambda_{1}(s, m)$ and $\nu_{1}=\nu_{1}(s, m)$ (see the dotted curves in Figure 8) having unique intersection point $P_{1}(m), Q_{1}(m)$ with the straight lines $\lambda_{1}=\bar{\lambda}_{1}, \nu_{1}=\bar{\nu}_{1}$, respectively. Note that $\lim _{m \rightarrow \infty} \nu_{1}(s, m)=0^{+}$for every $s \in(0,1)$. Then, $\lim _{m \rightarrow \infty} s\left(Q_{1}(m)\right)=0^{+}$, and the point $Q_{1}$ moves from $s=\frac{1}{2}$ (for $m=m_{\nu}^{-}$) to $s=0^{+}$(for $m=\infty$ ). Therefore, since $P_{1}$ moves to the right and $Q_{1}$ moves to the left, there exists a unique value $\widetilde{m}$ such that $\widetilde{s}=s\left(Q_{1}(\widetilde{m})\right)=s\left(P_{1}(\widetilde{m})\right)$. the pair $(\widetilde{s}, \widetilde{m})$ is the unique solution of the problem.

\section{SECOnd CASE.}

$$
\max \left\{m_{\lambda}^{-}, m_{\nu}^{-}\right\}=m_{\lambda}^{-},
$$

see Figure 9. We construct the curve $\nu_{1}=\nu_{1}\left(s, m_{\lambda}^{-}\right)$(see dashed curve in Figure 9 ). By increasing continuously the value of $m$ (from $m_{\lambda}^{-}$), the two curves ${ }_{275} \lambda_{1}=\lambda_{1}(s, m)$ and $\nu_{1}=\nu_{1}(s, m)$ intersect the lines $\lambda_{1}=\bar{\lambda}_{1}, \nu_{1}=\bar{\nu}_{1}$ at the points $P_{1}(m)$ and $Q_{1}(m)$, respectively (see dotted curves in Figure 9). Since $\lim _{m \rightarrow \infty} s\left(Q_{1}(m)\right)=0^{+}$, and the two points $P_{1}$ and $Q_{1}$ move one toward each other, there exists a unique solution to the identification problem.

\section{Applications}

\subsection{Numerical implementation of the identification method}

In order to apply the crack identification method illustrated in the previous section, the lower eigenvalues of the vibrating rod with different boundary conditions have to be determined. To do that a finite element model has been 
implemented. The rod has been discretized using a mesh $\left\{x_{0}=0<x_{1}<\ldots<\right.$

285

every $i=0, \ldots, N$. The corresponding eigenfunctions have been approximated by means of piecewise-linear continuous functions in $[0,1]$. The dimension $N$ of the finite element model has been chosen large enough to made the modelling errors on the lower eigenvalues negligible.

290 2.1 and 2.2 presented in Section 3. The application of procedure requires the determination of the $\lambda$-s curves for continuously varying values of the parameter $m$. Details on the numerical procedure can be found in [29].

The identification algorithm has been implemented on a computer with an dard numerical code written in Octave environment has been used to solve the discrete eigenvalue problem and to build the reconstruction procedure.

\subsection{Results of numerical tests}

The identification algorithm has been applied to a large variety of rod profiles, with different intensity and position of the point mass, and under elastically restrained end conditions having different stiffness. The results presented here correspond to rods with (normalized) cross-sectional area $a=a(x), x \in[0,1]$, given by

$$
a(x)=0.8(x(x-1)+1)
$$

The approximating eigenvalue problem was defined by dividing the interval $[0,1]$ into 200 equally spaced finite elements. The choice of the mesh-size guarantees negligible errors on the estimate of the first frequency of the rod.

Tables 2 to 4 quote the identification results corresponding to the scenario devised in Theorem 2.1. The identification algorithm was tested on twelve positions of the concentrated mass, and for each of them the intensity $m$ (simulating the presence of a crack) was taken equal to $0.001,0.01$, and 0.10 . Three different values of the masses located at the ends of the $\operatorname{rod} \mathcal{M}=0.01$ (Table 2), $\mathcal{M}=0.20$ (Table 3 ), and $\mathcal{M}=0.50$ (Table 4 ), simulating the elastically 
restrained support, were considered. These values have been chosen so that the first resonant frequency of the undamaged rod is equal to $97 \%, 57 \%, 33 \%$ of the are encountered when the conditions of the problem fill those corresponding to the First Case, e.g., exactly one in $(0,1 / 2)$ and exactly one in $(1 / 2,1)$. It is clear that one of them is the true physical solution, while the second one is the spurious or mathematical solution. This latter solution is characterized in 320 Tables 2-4 by large discrepancy with the actual values of the parameters $s$ and $m$. Similarly, for conditions corresponding to the Second Case in Theorem 2.1 (both for subcases a) and b)), only one solution is found by the identification algorithm. It is worth noting that in all the cases studied the transition between the First Case and the Second Case occurs near $s \simeq 0.66$. In order to underline improved in a neighborhood of $s \simeq 0.66$.

Summing up, numerical results lead to the conjecture that the most favorable conditions expressed in Table 1 hold true, that is, the knowledge of the first natural frequency of the ER-ER and ER-free rod is enough to determine exactly 330 the Second Case of Theorem 2.1, respectively.

Some identification results corresponding to the situation described in Theorem 2.2 are presented in Table 5. In this case, given the symmetry of the problem, only five positions of the concentrated mass have been considered, 335 always in the left half span of the rod. Here, a unique solution, as theoretically predicted by Theorem 2.2, is encountered. Again, the agreement between identified and actual values of the damage parameters is very good.

Once the point mass intensity $m$ and its location $s$ have been identified, the 
crack depth can be determined by means of the explicit relationship between the crack depth and the stiffness $K$. Considering a rod with rectangular crosssection, of side $B=$ const, height $H(x)=H\left(x_{0}\right) a(x)$ and area $\widehat{A}(x)=B H(x)$, which contains a pair of symmetric open edge cracks, each with front parallel to the side $B$, at the cross-section of (dimensionless) abscissa $s$. Denoting by $\frac{d}{2}$ the depth of each side crack, the stiffness $\widehat{K}$ of the elastic spring simulating the damage is expressed as

$$
\widehat{K}=\frac{E \widehat{A}(s)}{L \delta_{l}\left(\nu_{P} ; \alpha\right)},
$$

where (see [39])

$$
\begin{array}{r}
\delta_{l}\left(\nu_{P} ; \alpha\right)=2 \frac{H\left(z_{d}\right)}{L}\left(1-\nu_{P}^{2}\right)\left(0.7314 \alpha^{8}-1.0368 \alpha^{7}+0.5803 \alpha^{6}+1.2055 \alpha^{5}-\right. \\
\left.-1.0368 \alpha^{4}+0.2381 \alpha^{3}+0.9852 \alpha^{2}\right), \quad(7
\end{array}
$$

being $\alpha=\frac{d}{H(s)}$ the crack ratio and $\nu_{P}$ the Poisson ratio. Therefore, from the identified values of $m$ and $s$, it is possible, first, to determine $\delta_{l}\left(\nu_{P} ; \alpha\right)$ by

$$
\delta_{l}=m \frac{H(s)}{H\left(x_{0}\right)}
$$

and, next, the crack depth $d$ by inverting equation (76) with respect to $\alpha$. For usual values of $\nu_{P}$ (e.g., $\nu_{P} \simeq 0.3$ ), the function $\delta_{l}=\delta_{l}\left(\nu_{P} ; \cdot\right)$ is always uniquely invertible in the interval $\alpha \in[0,1]$, but the interval in which expression (76) is accurate is usually smaller. As an example, let us consider a steel rod of length $L=600 \mathrm{~mm}, H\left(x_{0}\right)=40 \mathrm{~mm}$, and with parabolic profile given by (74). Table 6 shows the dimensional position and crack depth corresponding to the dimensionless crack location and mass values considered in the identification cases collected in Table 5.

\subsection{Results with simulated experimental errors}

Tables 2 to 5 collect the results of identification in absence of errors on the data. In order to test the robustness of the algorithm, several selected cases have been analyzed in which experimental errors were simulated. For the 
selected cases, the fundamental eigenvalue $\lambda_{1}$ of the problem (8)-(12) and the first positive eigenvalue $\nu_{1}$ of the problem (24)-(28) were perturbed as

$$
\begin{aligned}
& \left(\lambda_{1}\right)^{\text {pert }}=\lambda_{1}\left(1+\tau_{1}\right)^{2}, \\
& \left(\nu_{1}\right)^{\text {pert }}=\nu_{1}\left(1+\tau_{2}\right)^{2},
\end{aligned}
$$

where $\tau_{1}$ and $\tau_{2}$ are real random Gaussian variables with zero mean and standard deviation $\sigma=3,33 \times 10^{-4}$. The maximum error considered with this random distribution of errors of the noisy data is $\pm 0.1 \%$ of the theoretical natural frequency $f$ (expressed in Hertz) measured in absence of errors.

For each case considered, a Monte Carlo simulation on a population of 100 samples has been carried out. The moderate number of samples used in the calculations is due to the huge computational cost of the complete analysis. Table 7 shows the statistical properties of the results of identification. All the showed results correspond to cases which meet the conditions described in Theorem 2.2.

The cases considered here correspond to mass values of $m=0.01$ and $m=$ 0.015. For these cases, the differences between the theoretical results and the mean values of the estimations are small for the position and moderate for the mass. However, for smaller values of $m$, the considered random errors could be higher than the variations of frequency promoted by the presence of crack (represented by the point mass), and the proposed constructive algorithm needs very long calculation time to find a solution, if it exists.

\section{Proof of auxiliary propositions}

The proof of Proposition 3.3 and Proposition 3.4 is based on some preliminary results.

Lemma 5.1. The eigenfunction $w_{1}$ associated to the eigenvalue $\lambda_{1}$ of (30)-(34) has exactly one simple zero in $(0,1)$. 
Lemma 5.2. The eigenfunction $y_{1}$ associated to the eigenvalue $\mu_{1}$ of (37)-(41) does not vanish in $[0,1)$.

It can be shown that a proof of the above statements can be obtained by extending the classical Sturm-Liouville approach (which holds for $m=0$ and $\mathcal{M}=0$ ), see, for example, [36]. Without going into the details, we recall that we found convenient to work directly on the original eigenvalue problem for the elastically-restrained cracked rod and, then, transfer the results to the equivalent eigenvalue problem with the point mass $m$ located at the damaged cross-section and the point mass $\mathcal{M}$ concentrated at each elastically restrained end.

Proposition 5.3. Let $(\lambda, w)$ be an eigenpair of $(30)-(34)$, for $m \in(0, \infty)$ and $s \in(0,1)$. We have

$$
\begin{aligned}
& \frac{\partial \lambda}{\partial s}=-\lambda \frac{m w(s)\left(w^{\prime}\left(s^{+}\right)+w^{\prime}\left(s^{-}\right)\right)}{\mathcal{M}\left(w^{2}(0)+w^{2}(1)\right)+m w^{2}(s)+\int_{0}^{1} b w^{2}}, \\
& \frac{\partial \lambda}{\partial m}=-\lambda \frac{w^{2}(s)}{\mathcal{M}\left(w^{2}(0)+w^{2}(1)\right)+m w^{2}(s)+\int_{0}^{1} b w^{2}},
\end{aligned}
$$

where $w(s)=w(x=s ; s, m), w^{\prime}\left(s^{+}\right)=\lim _{x_{0} \rightarrow s^{+}}\left(\left.\frac{d w(x ; s, m)}{d x}\right|_{x=x_{0}}\right), w^{\prime}\left(s^{-}\right)=$ $\lim _{x_{0} \rightarrow s^{-}}\left(\left.\frac{d w(x ; s, m)}{d x}\right|_{x=x_{0}}\right)$.

Let $(\mu, y)$ be an eigenpair of $(37)-(41)$, for $m \in(0, \infty)$ and $s \in(0,1)$. We have

$$
\begin{aligned}
\frac{\partial \mu}{\partial s} & =-\mu \frac{m y(s)\left(y^{\prime}\left(s^{+}\right)+y^{\prime}\left(s^{-}\right)\right)}{\mathcal{M} y^{2}(0)+m y^{2}(s)+\int_{0}^{1} b y^{2}}, \\
\frac{\partial \mu}{\partial m} & =-\mu \frac{y^{2}(s)}{\mathcal{M} y^{2}(0)+m y^{2}(s)+\int_{0}^{1} b y^{2}} .
\end{aligned}
$$

Proof. A proof can be obtained by adapting the arguments of the proof of Proposition 4.1 in [29].

Basing on the above properties of the eigenpairs $\left(\lambda_{1}, w_{1}\right),\left(\mu_{1}, y_{1}\right)$, and following the lines of the proof of Proposition 5.1 in [29], one can derive a proof of Proposition 3.3. We omit the details.

We conclude this section with a proof of Proposition 3.4. 
Proof of Proposition 3.4. Statements i) and ii) can be proved by following essentially the same lines. We present a detailed proof of i). The proof is by contradiction. Let us assume that there exists $\widetilde{s} \in\left(0, \frac{1}{2}\right)$ such that $\frac{d \lambda_{1}}{d s}(\widetilde{s})=0$, where $\frac{d \lambda_{1}}{d s}$ is given in (80) (for $\lambda=\lambda_{1}$ and $w=w_{1}$ ). If $w_{1}(\widetilde{s} ; \widetilde{s}, m)=0$, then, by Proposition 3.3 (point iv), $\widetilde{s}=\frac{1}{2}$, a contradiction with the assumption that $\widetilde{s} \in\left(0, \frac{1}{2}\right)$. Then, by $(80)$, it follows that

$$
w_{1}^{\prime}\left(\widetilde{s}^{+}\right)+w_{1}^{\prime}\left(\widetilde{s}^{-}\right)=0
$$

By (33) and (84) we have

$$
\begin{gathered}
b(\widetilde{s}) w_{1}^{\prime}\left(\widetilde{s}^{+}\right)=-\lambda_{1}(\widetilde{s}) \frac{m}{2} w_{1}(\widetilde{s}), \\
b(\widetilde{s}) w_{1}^{\prime}\left(\widetilde{s}^{-}\right)=\lambda_{1}(\widetilde{s}) \frac{m}{2} w_{1}(\widetilde{s}),
\end{gathered}
$$

where $w_{1}(\widetilde{s})=w_{1}(x=\widetilde{s} ; \widetilde{s}, m)$. Then, the restriction $\left.w_{1}\right|_{(0, \widetilde{s})},\left.w_{1}\right|_{(\widetilde{s}, 1)}$ of the function $w_{1}$ to the sub-interval $(0, \widetilde{s})$ and $(\widetilde{s}, 1)$, respectively - which will be denoted in the sequel by the same symbol $w_{1}$ to simplify the notation - satisfies separately the two eigenvalue problems

$$
\left\{\begin{array}{l}
\left(b w_{1}^{\prime}\right)^{\prime}+\lambda_{1}(\widetilde{s}) b w_{1}=0, \quad x \in(0, \widetilde{s}), \\
b(0) w_{1}^{\prime}(0)=-\lambda_{1}(\widetilde{s}) \mathcal{M} w_{1}(0), \\
b(\widetilde{s}) w_{1}^{\prime}(\widetilde{s})=\lambda_{1}(\widetilde{s}) \frac{m}{2} w_{1}(\widetilde{s})
\end{array}\right.
$$

and

$$
\left\{\begin{array}{l}
\left(b w_{1}^{\prime}\right)^{\prime}+\lambda_{1}(\widetilde{s}) b w_{1}=0, \quad x \in(\widetilde{s}, 1), \\
b(1) w_{1}^{\prime}(1)=\lambda_{1}(\widetilde{s}) \mathcal{M} w_{1}(1), \\
b(\widetilde{s}) w_{1}^{\prime}(\widetilde{s})=-\lambda_{1}(\widetilde{s}) \frac{m}{2} w_{1}(\widetilde{s}) .
\end{array}\right.
$$

It should be noticed that the eigenvalue problem (87)-(89) (respectively (90)(92)) describes the free longitudinal vibration of a rod carrying a point mass $\mathcal{M}$ at $x=0$ and a point mass $\frac{m}{2}$ at $x=\widetilde{s}$ (respectively, $\frac{m}{2}$ at $x=\widetilde{s}$ and $\mathcal{M}$ at $x=1$ for (90)-(92)). Then, it is well-known that the first eigenfunction of (87)(89) does not vanish in $[0, \widetilde{s}]$ and it is associated to the vanishing eigenvalue 
(similarly for the first eigenfunction of (90)-(92)). The vanishing eigenvalue corresponds to the rigid-body motion of the rod. Since $w_{1}(\widetilde{s}) \neq 0$ and $w_{1}$ has exactly one zero in $(0,1)$ (see Lemma 5.1 ), the function $w_{1}$ vanishes either in $(0, \widetilde{s})$ or in $(\widetilde{s}, 1)$. In every case, the restriction $\left.w_{1}\right|_{(\widetilde{s}, 1)}$ in the first case, or the restriction $\left.w_{1}\right|_{(0, \widetilde{s})}$ in the second case, is of one sign in the corresponding sub-interval. Therefore, either the restriction $\left.w_{1}\right|_{(0, \widetilde{s})}$ or the restriction $\left.w_{1}\right|_{(\widetilde{s}, 1)}$ coincides with the eigenfunction associated to the vanishing eigenvalue, that is $\lambda_{1}(\widetilde{s})=0$, which is a contradiction since $\lambda_{1}(\widetilde{s})>0$. Then $\frac{d \lambda_{1}}{d s}(s) \neq 0$ in $\left(0, \frac{1}{2}\right)$.

Finally, since $\lambda_{1}(s) \leq \lambda_{1}^{U}$ in $[0,1], \lambda_{1}\left(0, \frac{1}{2}\right)=\lambda_{1}^{U}, \lambda_{1}(0)<\lambda_{1}^{U}\left(\right.$ since $w_{1}(0) \neq$ $0)$ and

$$
\frac{d \lambda_{1}}{d s}(0)=\frac{\lambda_{1}^{2}(0) m(m+\mathcal{M}) w_{1}^{2}(0)}{b(0)\left(\mathcal{M}\left(w_{1}^{2}(0)+w_{1}^{2}(1)\right)+m w_{1}^{2}(0)+\int_{0}^{1} b w_{1}^{2}\right)}>0,
$$

the function $\lambda_{1}=\lambda_{1}(s)$ is an increasing function of $s$ in $\left(0, \frac{1}{2}\right)$, and the proof of the statement i) is complete.

\section{Conclusions}

Most of the results on identification of an open crack in a longitudinally

vibrating rod based on a suitable pair of resonant frequencies applies only when the boundary conditions are ideal, that is either for supported or free end conditions.

In this paper we have removed this limitation and we have considered the diagnostic problem for a rod under elastically restrained (ER) end conditions.

${ }_{15}$ Two specific problems have been studied in detail, namely the identification using the first (positive) natural frequency of the rod either under ER-ER and ER-free or under ER-ER and free-free boundary conditions.

For these cases we have set a constructive identification method. Uniqueness and non-uniqueness, as well as the stability of the procedure to errors on the input data have been discussed with reference both to general properties of 
the inverse problem and to results coming from numerical applications of the diagnostic technique.

As a final remark, we recall that the present analysis has been developed under the assumption that the undamaged configuration of the rod is completely

\section{Notation}

The following main symbols are used in this paper:

$\widehat{A}(z)$, cross-sectional area as a function of the abscissa $z, z \in$ $[0, L]$;

440 $x, x \in[0,1]$

$a(x)$, normalized cross-sectional area;

$b(x)$, inverse of the normalized cross-sectional area;

$B$, constant width of the rectangular cross-section of the rod;

${ }_{445} d, \alpha$, total crack length and crack ratio, respectively;

$E$, Young's modulus of the material; 
$\widehat{h}, h$, stiffness of an elastic end spring, and its normalized value, respectively;

$H(x)$, height of the cross-section of the rod;

450

$\widehat{K}, K$, stiffness of the longitudinal elastic spring modelling the crack, and its normalized value, respectively;

$L$, length of the rod;

$m$, intensity of a point mass internal to the rod segment;

$\mathcal{M}$, intensity of a point mass located at one end of the rod;

455

$N$, dimension of the finite element model;

$t(x), u(x), \widehat{u}(z), v(x), w(x), y(x), \zeta(x)$, longitudinal vibration of the perturbed rod (eigenfunction);

$t^{U}(x), u^{U}(x), v^{U}(x), w^{U}(x), y^{U}(x)$, longitudinal vibration of the unperturbed rod (eigenfunction);

$x, z$, longitudinal abscissa and normalized longitudinal abscissa, respectively;

$z_{d}, s$, abscissa of the cracked cross-section, and its normalized value, respectively;

$\gamma$, volume mass density;

$\delta_{l}$, normalized flexibility (inverse of stiffness) promoted by the crack;

$\lambda, \mu, \nu$, eigenvalues of the perturbed rod;

$\lambda^{U}, \mu^{U}, \nu^{U}$, eigenvalues of the unperturbed rod;

$\nu_{P}$, Poisson's ratio;

470

$\tau_{1}, \tau_{2}$, real random Gaussian variables;

$\omega$, circular frequency. 


\section{References}

[1] R.D. Adams, P. Cawley, C.J. Pye, B.J. Stone, A vibration technique for non-destructively assessing the integrity of structures, 475 Journal of Mechanical Engineering Science 20 (1978) 93-100.

[2] T.G. Chondros, A.D. Dimarogonas, Identification of cracks in welded joints of complex structures, Journal of Sound and Vibration 69(4) (1980) 531-538.

[3] G. Hearn, R.B. Testa, Modal analysis for damage detection in ${ }_{480} \quad$ structures, Journal of Structural Engineering ASCE 117 (1991) 3042-3063.

[4] R.Y. Liang, J. Hu, F. Choy, Theoretical study of crack-induced eigenfrequency changes on beam structures, Journal of Engineering Mechanics ASCE 118 (1992) 384-396.

${ }_{485}$ [5] F. Vestroni, D. Capecchi, Damage evaluation in cracked vibrating beams using experimental frequencies and finite element model, Journal of Vibration and Control 2(1) (1996) 69-86.

[6] D. Capecchi, F. Vestroni, Monitoring of structural systems by using frequency data, Earthquake Engineering and Structural Dynamics 28 (2000) 447-461.

[7] M.N. Cerri, F. Vestroni, Detection of damage in beams subjected to diffuse cracking. Journal of Sound and Vibration 234(2) (2000) 259-276. 
[8] T.D. Chaudhari, S.K. Maiti, A study of vibration of geometrically segmented beams with and without crack, International Journal of Solids and Structures 37 (2000) 761-779.

[9] F. Vestroni, D. Capecchi, Damage detection in beam structures based on frequency measurements, Journal of Engineering Mechanics ASCE 126 (2000) 761-768.

${ }_{500}$ [10] S. Chinchalkar, Determination of crack location in beams using natural frequencies, Journal of Sound and Vibration 247(3) (2001) 417-429.

[11] J.K. Sinha, M.I. Friswell, S. Edwards, Simplified models for the location of cracks in beam structures using measured vibration data, Journal of Sound and Vibration 251 (2002) 13-38.

[12] A. Teughels, J. Maeck, G. De Roeck, Damage assessment by FE model updating using damage functions, Computer and Structures 80 (2002) 1869-1879.

[13] A. Morassi, Damage detection and generalized Fourier coefficients, Journal of Sound and Vibration 302 (2007) 229-259.

[14] A. Morassi, F. Vestroni (Eds.), Dynamic Methods for Damage Identification in Structures, CISM Courses and Lectures No. 499, Springer, Wien, Austria, 2008.

[15] L. Rubio, An efficient method for crack identification in sim${ }_{515}$ ply supported Euler-Bernoulli beams, Journal of Vibration and Acoustics 131 (2009) 051001. 
[16] M. Dilena, A. Morassi, Reconstruction method for damage detection in beams based on natural frequency and antiresonant frequency measurements, Journal of Engineering Mechanics ASCE 136(3) (2010) 329-344.

[17] A. Pau, A. Greco, F. Vestroni, Numerical and experimental detection of concentrated damage in a parabolic arch by measured frequency variations, Journal of Vibration and Control 17(4) (2011) 605-614.

${ }_{525}$ [18] A. Greco, A. Pau, Damage identification in Euler frames, Computer and Structures 92-93 (2012) 328-336.

[19] S. Caddemi, I. Caliò, The exact explicit dynamic stiffness matrix of multi-cracked Euler-Bernoulli beam and applications to damaged frame structures, Journal of Sound and Vibration 332 (2013) 3049-3063.

[20] S. Caddemi, I. Caliò, Exact reconstruction of multiple concentrated damages on beams, Acta Mechanica 225 (2014) 31373156.

[21] N.T. Khiem, L.K. Toan, A novel method for crack detection in beam-like structures by measurements of natural frequencies, Journal of Sound and Vibration 333 (2014) 4084-4103.

[22] G.M.L. Gladwell, Inverse problems in vibration, Second edition, Kluwer Academic Publishers, Dordrecht, The Netherlands, 2004. 
[23] L.B. Freund, G. Herrmann, Dynamic fracture of a beam or plate in plane bending, Journal of Applied Mechanics 76-APM15 (1976) 112-116.

[24] E. Cabib, L. Freddi, A. Morassi, D. Percivale, Thin notched beams. Journal of Elasticity 64(2/3) (2001) 157-178.

[25] Y. Narkis, Identification of crack location in vibrating simply supported beams, Journal of Sound and Vibration 172 (1994) 549-558.

[26] A. Morassi, Identification of a crack in a rod based on changes in a pair of natural frequencies, Journal of Sound and Vibration 242 (2001) 577-596.

[27] M. Dilena, A. Morassi, The use of antiresonances for crack detection in beams, Journal of Sound and Vibration 276 (2004) $195-214$

[28] L. Rubio, J. Fernández-Sáez, A. Morassi, The full nonlinear crack detection problem in uniform vibrating rods, Journal of Sound and Vibration 339 (2015) 99-111.

[29] L. Rubio, J. Fernández-Sáez, A. Morassi, Identification of an open crack in a non-uniform rod by two frequency data, International Journal of Solids and Structures 75-76 (2015) 61-80.

[30] W.T. Springer, K.L. Lawrence, T.J. Lawley, Damage assessment based on the structural frequency-response function, $E x$ perimental Mechanics 28 (1988) 34-37. 
[31] C. Davini, F. Gatti, A. Morassi, A damage analysis of steel beams, Meccanica 28 (1993) 27-37.

[32] T.G. Chondros, A.D. Dimarogonas, J. Yao, A consistent cracked bar vibration theory, Journal of Sound and Vibration 200 (1997) 303-313.

[33] G. Biscontin, A. Morassi, P. Wendel, Asymptotic separation of the spectrum in notched rods, Journal of Vibration and Control 4 (1998) 237-251.

570 [34] G.M.L. Gladwell, A. Morassi, Estimating damage in a rod from changes in node positions, Inverse Problems in Engineering 7 (1999) 215-233.

[35] N.T. Khiem, P.T. Hang, L.K. Toan, Crack detection in pile by measurements of frequency response function, Nondestructive Testing and Evaluation 31 (2016) 122-141.

[36] R. Courant, D. Hilbert Methods of Mathematical Physics, vol. I, First English Edition, Interscience Publishers, Inc., NewYork, 1966.

[37] H.F. Weinberger, Variational Methods for Eigenvalue Approximation, Regional Conference Series in Applied Mathematics, Society for Industrial and Applied Mathematics, Philadelphia, 1974. 
[38] G. Borg, Eine Umkehrung der Sturm-Liouvilleschen Eigenwertaufgabe. Bestimmung der Differentialgleichung durch die Eigenwerte. Acta Mathematica 78 (1946) 1-96.

[39] R. Ruotolo, C. Surace, Natural frequencies of a bar with multiple cracks, Journal of Sound and Vibration 272 (2004) 301-316. 


\section{Table Captions}

Table 1. Theorem 2.1: Number of solutions $\sharp$ expected for the crack identification problem.

Table 2. Identification of the mass intensity $m$ and position $s$ in a rod with (normalized) cross-sectional area given by (74) and $\mathcal{M}=0.01$. Cases (and subcases, in brackets) corresponding to Theorem 2.1. Percentage errors: $e_{m}=100 \times\left(m_{e s t}-m\right) / m, e_{s}=$ $100 \times\left(s_{\text {est }}-s\right) / s$.

Table 3. Identification of the mass intensity $m$ and position $s$ in a rod with (normalized) cross-sectional area given by (74) and $\mathcal{M}=$ 0.2. Cases (and subcases, in brackets) corresponding to Theorem 2.1. Percentage errors: $e_{m}=100 \times\left(m_{\text {est }}-m\right) / m, e_{s}=100 \times\left(s_{\text {est }}-\right.$ $600 s) / s$.

Table 4. Identification of the mass intensity $m$ and position $s$ in a rod with (normalized) cross-sectional area given by (74) and $\mathcal{M}=$ 0.5. Cases (and subcases, in brackets) corresponding to Theorem 2.1. Percentage errors: $e_{m}=100 \times\left(m_{\text {est }}-m\right) / m, e_{s}=100 \times\left(s_{\text {est }}-\right.$ $605 s) / s$.

Table 5. Theorem 2.2: identification of the mass intensity $m$ and position $s$ in a rod with (normalized) cross-sectional area given by (74). Percentage errors: $e_{m}=100 \times\left(m_{\text {est }}-m\right) / m, e_{s}=100 \times\left(s_{\text {est }}-\right.$ $s) / s$.

${ }_{610}$ Table 6. Relationship between mass $m$ and crack depth $d$ for different crack positions $s$ in a rod with (normalized) cross-sectional 
area given by (74). $L=600 \mathrm{~mm}, H\left(x_{0}\right)=40 \mathrm{~mm}$; the quantities $s$, $H(s), d$ are expressed in $\mathrm{mm}$.

Table 7. Identification of the mass intensity $m$ and position $s$ with random errors in the frequency data in a rod with (normalized) cross-sectional area given by (74). Cases corresponding to Theorem 2.2 and perturbed data as in (78)-(79). Percentage errors: $e_{m}=$ $100 \times\left(m_{e s t}-m\right) / m, e_{s}=100 \times\left(s_{e s t}-s\right) / s$. 


\section{Figure Captions}

${ }_{620}$ Figure 1. ER-ER cracked rod.

Figure 2. ER-free cracked rod.

Figure 3. Equivalent problem to the ER-ER cracked rod in Figure 1 .

Figure 4. Equivalent problem to the ER-free cracked rod in Figure 6252.

Figure 5. Proof of Theorem 2.1: first case.

Figure 6. Proof of Theorem 2.1: second case - subcase a).

Figure 7. Proof of Theorem 2.1: second case - subcase b).

Figure 8. Proof of Theorem 2.2: first case.

${ }_{630}$ Figure 9. Proof of Theorem 2.2: second case. 
Table 1: Theorem 2.1: Number of solutions $\sharp$ expected for the crack identification problem.

\begin{tabular}{ccc}
\hline Case & Number of solutions $\sharp$ \\
& $s \in\left(0, \frac{1}{2}\right)$ & $s \in\left(\frac{1}{2}, 1\right)$ \\
\hline First & $\geq 1$ & 1 \\
Second, subcase a) & $\geq 0$ & 1 \\
Second, subcase b) & 0 & 1 \\
\hline
\end{tabular}


Table 2: Identification of the mass intensity $m$ and position $s$ in a rod with (normalized) crosssectional area given by (74) and $\mathcal{M}=0.01$. Cases (and subcases, in brackets) corresponding to Theorem 2.1. Percentage errors: $e_{m}=100 \times\left(m_{\text {est }}-m\right) / m, e_{s}=100 \times\left(s_{\text {est }}-s\right) / s$.

\begin{tabular}{|c|c|c|c|c|c|c|}
\hline \multirow[b]{2}{*}{$\mathrm{m}$} & \multirow[b]{2}{*}{$\mathrm{s}$} & \multicolumn{2}{|c|}{ First solution } & \multicolumn{2}{|c|}{ Second solution } & \multirow[b]{2}{*}{ Case } \\
\hline & & $e_{s}$ & $e_{m}$ & $e_{s}$ & $e_{m}$ & \\
\hline \multirow{12}{*}{0.001} & 0.05 & 20.91 & 0.19 & 1211.35 & 332.58 & 1 \\
\hline & 0.20 & 3.04 & 0.35 & 219.54 & 215.10 & 1 \\
\hline & 0.35 & -0.30 & -0.54 & 69.79 & 122.30 & 1 \\
\hline & 0.45 & -0.42 & -0.71 & 20.27 & 37.05 & 1 \\
\hline & 0.65 & -72.24 & -73.93 & -0.08 & 0.54 & 1 \\
\hline & 0.66 & 0.01 & 0.99 & & & $2(\mathrm{a})$ \\
\hline & 0.67 & -0.06 & 0.77 & & & $2(\mathrm{a})$ \\
\hline & 0.68 & -0.19 & 0.31 & & & $2(\mathrm{a})$ \\
\hline & 0.69 & -0.11 & 0.73 & & & $2(\mathrm{a})$ \\
\hline & 0.70 & -0.15 & 0.62 & & & $2(\mathrm{a})$ \\
\hline & 0.85 & -0.35 & 0.68 & & & 2 (b) \\
\hline & 0.95 & -0.47 & 0.07 & & & $2(\mathrm{~b})$ \\
\hline \multirow{12}{*}{0.010} & 0.05 & 3.04 & -0.09 & 1212.39 & 329.39 & 1 \\
\hline & 0.20 & -0.83 & -0.51 & 219.43 & 251.41 & 1 \\
\hline & 0.35 & -0.98 & -1.04 & 69.77 & 121.14 & 1 \\
\hline & 0.45 & -0.94 & -1.39 & 19.95 & 36.05 & 1 \\
\hline & 0.65 & -78.48 & -74.78 & -0.22 & 0.03 & 1 \\
\hline & 0.66 & -0.22 & 0.09 & & & $2(\mathrm{a})$ \\
\hline & 0.67 & -0.23 & 0.08 & & & $2(\mathrm{a})$ \\
\hline & 0.68 & -0.24 & 0.09 & & & $2(\mathrm{a})$ \\
\hline & 0.69 & -0.26 & 0.08 & & & $2(\mathrm{a})$ \\
\hline & 0.70 & -0.27 & 0.08 & & & $2(\mathrm{a})$ \\
\hline & 0.85 & -0.40 & 0.04 & & & $2(\mathrm{~b})$ \\
\hline & 0.95 & -0.55 & -3.08 & & & $2(\mathrm{~b})$ \\
\hline \multirow{12}{*}{0.100} & 0.05 & -1.88 & -0.22 & 1237.35 & 322.13 & 1 \\
\hline & 0.20 & -1.03 & -0.57 & 221.96 & 234.24 & 1 \\
\hline & 0.35 & -1.00 & -1.02 & 71.15 & 116.54 & 1 \\
\hline & 0.45 & -1.00 & -1.42 & 19.92 & 34.14 & 1 \\
\hline & 0.65 & -73.41 & -73.84 & -0.23 & 0.01 & 1 \\
\hline & 0.66 & -93.35 & -76.74 & -0.24 & 0.01 & 1 \\
\hline & 0.67 & -0.25 & 0.00 & & & $2(\mathrm{a})$ \\
\hline & 0.68 & -0.26 & 0.00 & & & $2(\mathrm{a})$ \\
\hline & 0.69 & -0.27 & 0.00 & & & $2(\mathrm{a})$ \\
\hline & 0.70 & -0.28 & 0.01 & & & $2(\mathrm{a})$ \\
\hline & 0.85 & -0.41 & 0.00 & & & $2(\mathrm{~b})$ \\
\hline & 0.95 & -0.47 & 0.00 & & & $2(\mathrm{~b})$ \\
\hline
\end{tabular}


Table 3: Identification of the mass intensity $m$ and position $s$ in a rod with (normalized) crosssectional area given by (74) and $\mathcal{M}=0.2$. Cases (and subcases, in brackets) corresponding to Theorem 2.1. Percentage errors: $e_{m}=100 \times\left(m_{\text {est }}-m\right) / m, e_{s}=100 \times\left(s_{\text {est }}-s\right) / s$.

\begin{tabular}{|c|c|c|c|c|c|c|}
\hline \multirow[b]{2}{*}{$\mathrm{m}$} & \multirow[b]{2}{*}{$\mathrm{s}$} & \multicolumn{2}{|c|}{ First solution } & \multicolumn{2}{|c|}{ Second solution } & \multirow[b]{2}{*}{ Case } \\
\hline & & $e_{s}$ & $e_{m}$ & $e_{s}$ & $e_{m}$ & \\
\hline \multirow{12}{*}{0.001} & 0.05 & 1.75 & -0.28 & 1214.83 & 427.83 & 1 \\
\hline & 0.20 & 1.35 & -0.04 & 217.70 & 292.24 & 1 \\
\hline & 0.35 & -0.19 & -0.52 & 69.86 & 136.30 & 1 \\
\hline & 0.45 & -0.87 & -1.41 & 20.16 & 39.70 & 1 \\
\hline & 0.65 & -75.51 & -73.78 & -0.03 & 0.75 & 1 \\
\hline & 0.66 & -88.10 & -80.81 & -0.05 & & $2(\mathrm{a})$ \\
\hline & 0.67 & -0.20 & 0.20 & & & $2(\mathrm{a})$ \\
\hline & 0.68 & -0.12 & 0.63 & & & $2(\mathrm{a})$ \\
\hline & 0.69 & -0.18 & 0.43 & & & $2(\mathrm{a})$ \\
\hline & 0.70 & -0.12 & 0.81 & & & $2(\mathrm{a})$ \\
\hline & 0.85 & -0.33 & 0.97 & & & 2 (b) \\
\hline & 0.95 & -0.45 & 0.63 & & & $2(\mathrm{~b})$ \\
\hline \multirow{12}{*}{0.010} & 0.05 & -1.23 & -0.39 & 1217.49 & 425.54 & 1 \\
\hline & 0.20 & -1.01 & -0.75 & 218.43 & 291.86 & 1 \\
\hline & 0.35 & -0.93 & -1.15 & 69.76 & 134.84 & 1 \\
\hline & 0.45 & -0.98 & -1.57 & 19.93 & 38.91 & 1 \\
\hline & 0.65 & -78.93 & -78.73 & -0.20 & 0.09 & 1 \\
\hline & 0.66 & -92.98 & -81.26 & -0.24 & 0.01 & 1 \\
\hline & 0.67 & -0.24 & 0.03 & & & $2(\mathrm{a})$ \\
\hline & 0.68 & -0.25 & 0.05 & & & $2(\mathrm{a})$ \\
\hline & 0.69 & -0.26 & 0.05 & & & $2(\mathrm{a})$ \\
\hline & 0.70 & -0.28 & 0.02 & & & $2(\mathrm{a})$ \\
\hline & 0.85 & -0.41 & 0.00 & & & $2(\mathrm{~b})$ \\
\hline & 0.95 & -0.47 & 0.05 & & & $2(\mathrm{~b})$ \\
\hline \multirow{12}{*}{0.100} & 0.05 & -1.75 & -0.42 & 1234.29 & 397.2 & 1 \\
\hline & 0.20 & -1.02 & -0.74 & 221.08 & 272.85 & 1 \\
\hline & 0.35 & -1.00 & -1.17 & 69.75 & 125.73 & 1 \\
\hline & 0.45 & -0.99 & -1.53 & 19.92 & 37.20 & 1 \\
\hline & 0.65 & -73.88 & -73.63 & -0.23 & 0.00 & 1 \\
\hline & 0.66 & -90.26 & -80.55 & -0.24 & 0.00 & 1 \\
\hline & 0.67 & -0.25 & 0.01 & & & $2(\mathrm{a})$ \\
\hline & 0.68 & -0.26 & 0.00 & & & $2(\mathrm{a})$ \\
\hline & 0.69 & -0.27 & 0.00 & & & $2(\mathrm{a})$ \\
\hline & 0.70 & -0.28 & 0.01 & & & $2(\mathrm{a})$ \\
\hline & 0.85 & -0.41 & 0.00 & & & $2(\mathrm{~b})$ \\
\hline & 0.95 & -0.47 & 0.00 & & & $2(\mathrm{~b})$ \\
\hline
\end{tabular}


Table 4: Identification of the mass intensity $m$ and position $s$ in a rod with (normalized) crosssectional area given by (74) and $\mathcal{M}=0.5$. Cases (and subcases, in brackets) corresponding to Theorem 2.1. Percentage errors: $e_{m}=100 \times\left(m_{\text {est }}-m\right) / m, e_{s}=100 \times\left(s_{\text {est }}-s\right) / s$.

\begin{tabular}{|c|c|c|c|c|c|c|}
\hline \multirow[b]{2}{*}{$\mathrm{m}$} & \multirow[b]{2}{*}{$\mathrm{s}$} & \multicolumn{2}{|c|}{ First solution } & \multicolumn{2}{|c|}{ Second solution } & \multirow[b]{2}{*}{ Case } \\
\hline & & $e_{s}$ & $e_{m}$ & $e_{s}$ & $e_{m}$ & \\
\hline \multirow{12}{*}{0.001} & 0.05 & 6.83 & -0.12 & 1210.21 & 505.25 & 1 \\
\hline & 0.20 & 1.17 & -0.12 & 216.96 & 328.44 & 1 \\
\hline & 0.35 & -0.65 & -1.01 & 69.15 & 145.02 & 1 \\
\hline & 0.45 & -0.92 & -1.59 & 20.13 & 42.06 & 1 \\
\hline & 0.65 & -80.29 & -81.08 & -0.01 & 0.83 & 1 \\
\hline & 0.66 & -90.39 & -83.66 & -0.09 & 0.61 & 1 \\
\hline & 0.67 & -0.08 & 0.74 & & & $2(\mathrm{a})$ \\
\hline & 0.68 & -0.18 & 0.36 & & & $2(\mathrm{a})$ \\
\hline & 0.69 & -0.06 & 0.98 & & & $2(\mathrm{a})$ \\
\hline & 0.70 & -0.08 & 0.99 & & & $2(\mathrm{a})$ \\
\hline & 0.85 & -0.34 & 0.86 & & & 2 (b) \\
\hline & 0.95 & -0.47 & 0.07 & & & $2(\mathrm{~b})$ \\
\hline \multirow{12}{*}{0.010} & 0.05 & -0.02 & -0.51 & 1211.44 & 500.75 & 1 \\
\hline & 0.20 & -0.89 & -0.86 & 216.94 & 325.09 & 1 \\
\hline & 0.35 & -0.95 & -1.29 & 69.15 & 144.21 & 1 \\
\hline & 0.45 & -0.95 & -1.63 & 19.94 & 41.39 & 1 \\
\hline & 0.65 & -81.40 & -81.24 & -0.22 & 0.04 & 1 \\
\hline & 0.66 & -94.50 & -84.12 & -0.24 & 0.01 & 1 \\
\hline & 0.67 & -0.24 & 0.03 & & & $2(\mathrm{a})$ \\
\hline & 0.68 & -0.25 & 0.04 & & & $2(\mathrm{a})$ \\
\hline & 0.69 & -0.26 & 0.05 & & & $2(\mathrm{a})$ \\
\hline & 0.70 & -0.27 & 0.07 & & & $2(\mathrm{a})$ \\
\hline & 0.85 & -0.41 & 0.01 & & & $2(\mathrm{~b})$ \\
\hline & 0.95 & -0.47 & 0.04 & & & $2(\mathrm{~b})$ \\
\hline \multirow{12}{*}{0.100} & 0.05 & -1.69 & -0.60 & 1227.69 & 471.43 & 1 \\
\hline & 0.20 & -1.02 & -0.88 & 219.34 & 307.98 & 1 \\
\hline & 0.35 & -0.99 & -1.28 & 69.74 & 138.90 & 1 \\
\hline & 0.45 & -0.99 & -1.64 & 19.91 & 39.97 & 1 \\
\hline & 0.65 & -80.98 & -80.82 & -0.23 & 0.01 & 1 \\
\hline & 0.66 & -93.32 & -83.69 & -0.24 & 0.01 & 1 \\
\hline & 0.67 & -0.25 & 0.00 & & & $2(\mathrm{a})$ \\
\hline & 0.68 & -0.26 & 0.01 & & & $2(\mathrm{a})$ \\
\hline & 0.69 & -0.27 & 0.00 & & & $2(\mathrm{a})$ \\
\hline & 0.70 & -0.28 & 0.00 & & & $2(\mathrm{a})$ \\
\hline & 0.85 & -0.41 & 0.00 & & & $2(\mathrm{~b})$ \\
\hline & 0.95 & -0.47 & 0.01 & & & $2(\mathrm{~b})$ \\
\hline
\end{tabular}


Table 5: Theorem 2.2: identification of the mass intensity $m$ and position $s$ in a rod with (normalized) cross-sectional area given by (74). Percentage errors: $e_{m}=100 \times\left(m_{e s t}-m\right) / m$, $e_{s}=100 \times\left(s_{\text {est }}-\underline{s}\right) / s$.

\begin{tabular}{|c|c|c|c|c|c|c|c|}
\hline \multirow[b]{2}{*}{$\mathcal{M}$} & \multirow[b]{2}{*}{$\mathrm{s}$} & \multicolumn{2}{|c|}{$\mathrm{m}=0.001$} & \multicolumn{2}{|c|}{$\mathrm{m}=0.010$} & \multicolumn{2}{|c|}{$\mathrm{m}=0.100$} \\
\hline & & $e_{s}$ & $e_{m}$ & $e_{s}$ & $e_{m}$ & $e_{s}$ & $e_{m}$ \\
\hline \multirow{5}{*}{0.01} & 0.05 & 9.35 & 0.54 & 0.57 & -2.49 & 9.45 & 0.76 \\
\hline & 0.15 & -0.50 & 0.01 & -0.51 & 0.06 & -0.50 & 0.01 \\
\hline & 0.25 & -0.51 & 0.69 & -0.50 & 0.08 & -0.50 & 0.01 \\
\hline & 0.35 & -0.50 & 0.19 & -0.50 & 0.05 & -0.50 & 0.01 \\
\hline & 0.45 & -0.50 & 0.05 & -0.50 & 0.00 & -0.50 & 0.01 \\
\hline \multirow{5}{*}{0.20} & 0.05 & 8.87 & 1.73 & 9.37 & 1.25 & 9.45 & 1.26 \\
\hline & 0.15 & -0.52 & 0.33 & -0.50 & 0.08 & -0.50 & 0.00 \\
\hline & 0.25 & -0.50 & 0.09 & -0.50 & 0.02 & -0.50 & 0.01 \\
\hline & 0.35 & -0.50 & 0.19 & -0.50 & 0.05 & -0.50 & 0.01 \\
\hline & 0.45 & -0.50 & 0.05 & -0.50 & 0.00 & -0.50 & 0.01 \\
\hline \multirow{5}{*}{0.50} & 0.05 & 8.77 & 2.45 & 9.45 & 1.52 & 9.45 & 1.56 \\
\hline & 0.15 & -0.54 & 0.76 & -0.50 & 0.01 & -0.50 & 0.01 \\
\hline & 0.25 & -0.50 & 0.09 & -0.50 & 0.02 & -0.50 & 0.01 \\
\hline & 0.35 & -0.50 & 0.19 & -0.50 & 0.05 & -0.50 & 0.01 \\
\hline & 0.45 & -0.50 & 0.05 & -0.50 & 0.00 & -0.50 & 0.01 \\
\hline
\end{tabular}


Table 6: Relationship between mass $m$ and crack depth $d$ for different crack positions $s$ in a rod with (normalized) cross-sectional area given by (74). $L=600 \mathrm{~mm}, H\left(x_{0}\right)=40 \mathrm{~mm}$; the quantities $s, H(s), d$ are expressed in $\mathrm{mm}$.

\begin{tabular}{ccccc}
\hline & & $m=0.001 ; \alpha=0.09$ & $m=0.010 ; \alpha=0.29$ & $m=0.100 ; \alpha=0.82$ \\
\cline { 3 - 5 }$s$ & $H(s)$ & $d$ & $d$ & $d$ \\
\hline 30.00 & 33.52 & 3.04 & 9.63 & 27.32 \\
90.00 & 36.08 & 3.28 & 10.36 & 29.41 \\
150.00 & 38.00 & 3.45 & 10.91 & 30.97 \\
210.00 & 39.28 & 3.57 & 11.28 & 32.01 \\
270.00 & 39.92 & 3.63 & 11.47 & 32.54 \\
\hline
\end{tabular}


Table 7: Identification of the mass intensity $m$ and position $s$ with random errors in the frequency data in a rod with (normalized) cross-sectional area given by (74). Cases corresponding to Theorem 2.2 and perturbed data as in (78)-(79). Percentage errors: $e_{m}=100 \times\left(m_{\text {est }}-m\right) / m, e_{s}=100 \times\left(s_{\text {est }}-s\right) / s$.

\begin{tabular}{|c|c|c|c|c|c|c|}
\hline $\mathcal{M}$ & $\mathrm{m}$ & $\mathrm{s}$ & $e_{s}($ Mean $)$ & $s_{\text {est }}(S t d)$ & $e_{m}($ Mean $)$ & $m_{\text {est }}(S t d)$ \\
\hline \multirow{5}{*}{0.01} & 0.010 & 0.15 & -0.69 & 0.011212 & -0.86 & 0.000512 \\
\hline & & 0.35 & -1.12 & 0.013660 & -0.91 & 0.000577 \\
\hline & & & & & & \\
\hline & 0.015 & 0.15 & -0.98 & 0.007040 & -4.50 & 0.000535 \\
\hline & & 0.35 & -0.93 & 0.007855 & 2.74 & 0.000578 \\
\hline \multirow{5}{*}{0.20} & 0.010 & 0.15 & -1.33 & 0.010824 & 10.04 & 0.000781 \\
\hline & & 0.35 & -0.08 & 0.024021 & 4.31 & 0.000921 \\
\hline & & & & & & \\
\hline & 0.015 & 0.15 & -0.79 & 0.007671 & -1.22 & 0.000771 \\
\hline & & 0.35 & -0.79 & 0.019048 & -0.01 & 0.000974 \\
\hline
\end{tabular}




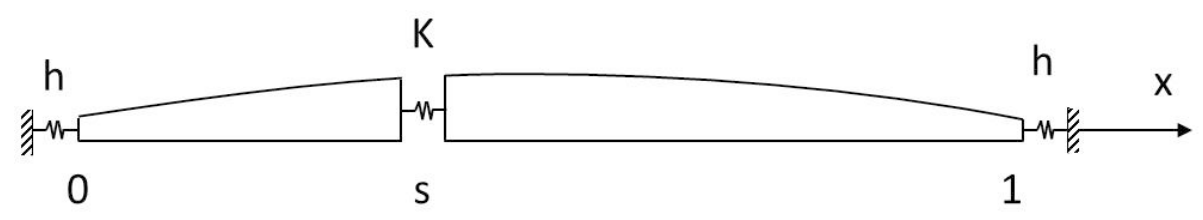

Figure 1: ER-ER cracked rod. 


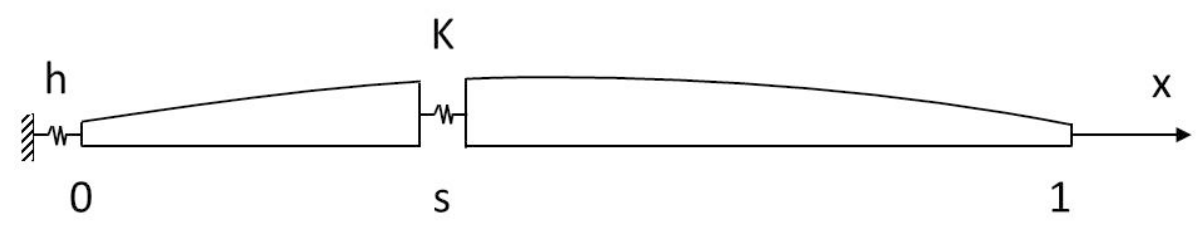

Figure 2: ER-free cracked rod. 


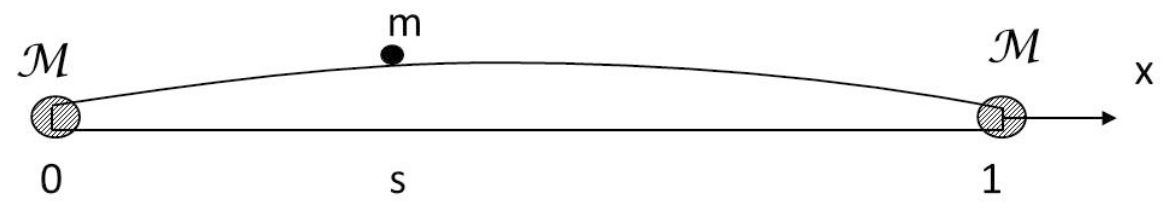

Figure 3: Equivalent problem to the ER-ER cracked rod in Figure 1. 


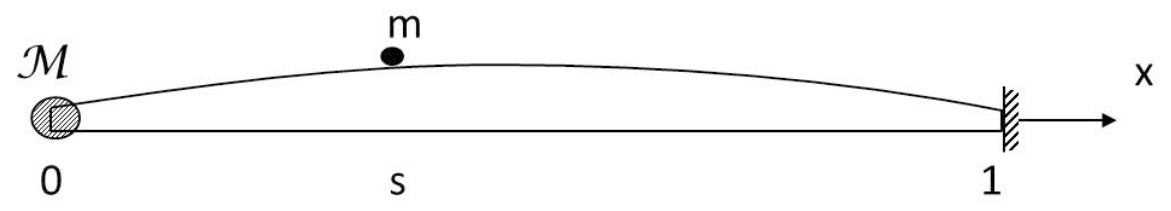

Figure 4: Equivalent problem to the ER-free cracked rod in Figure 2. 


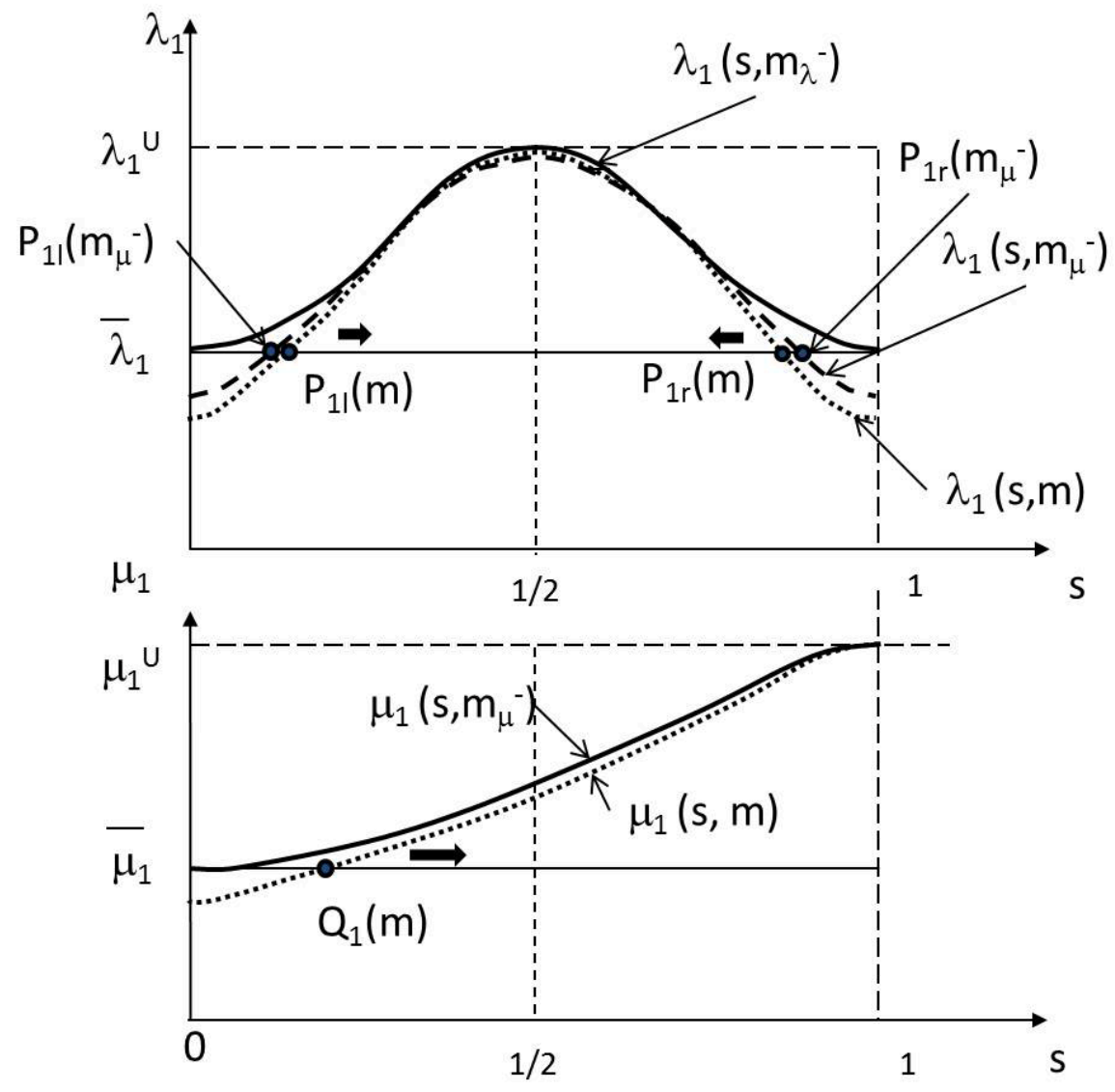

Figure 5: Proof of Theorem 2.1: first case. 


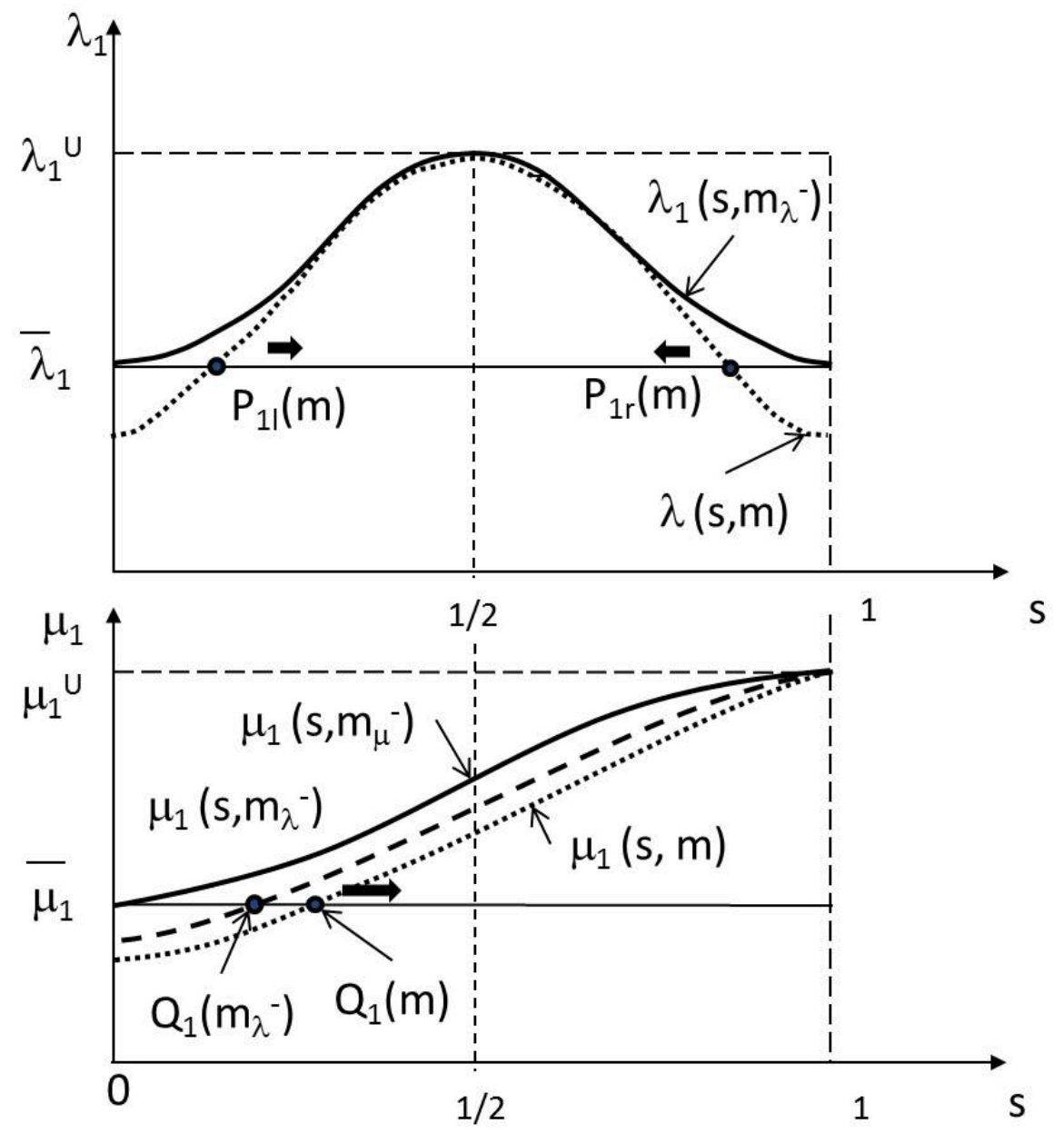

Figure 6: Proof of Theorem 2.1: second case - subcase a). 


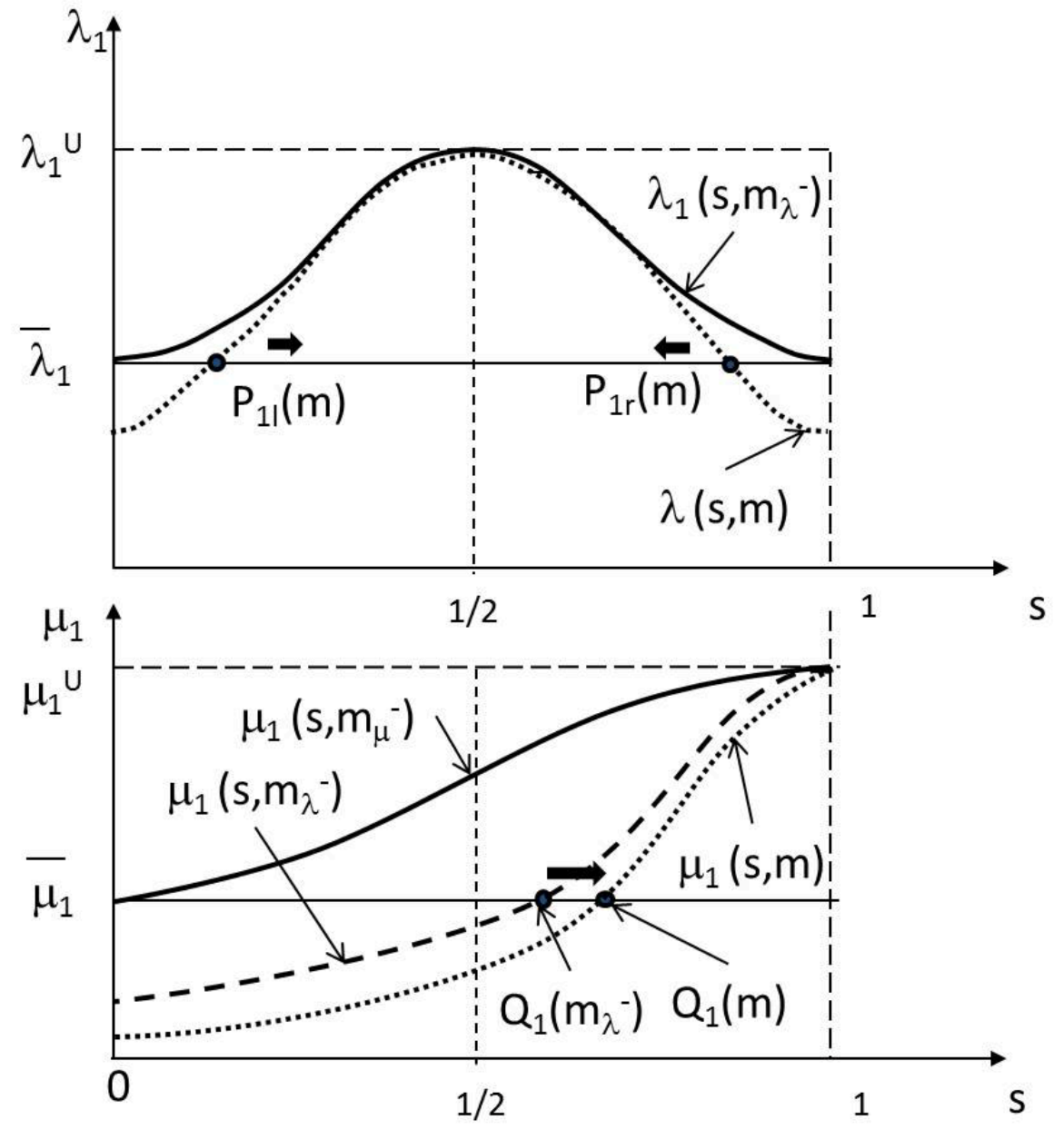

Figure 7: Proof of Theorem 2.1: second case - subcase b). 

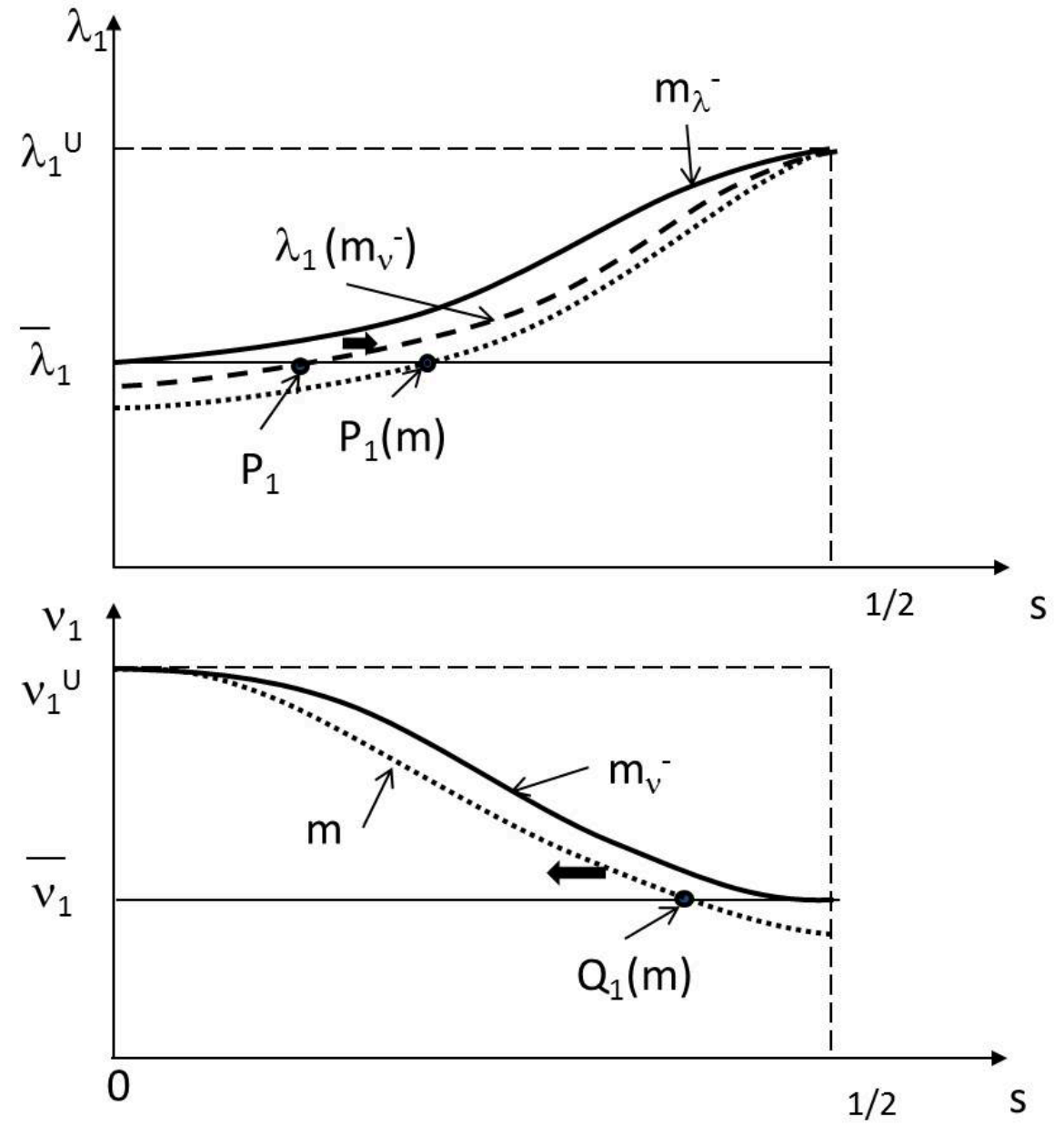

Figure 8: Proof of Theorem 2.2: first case. 

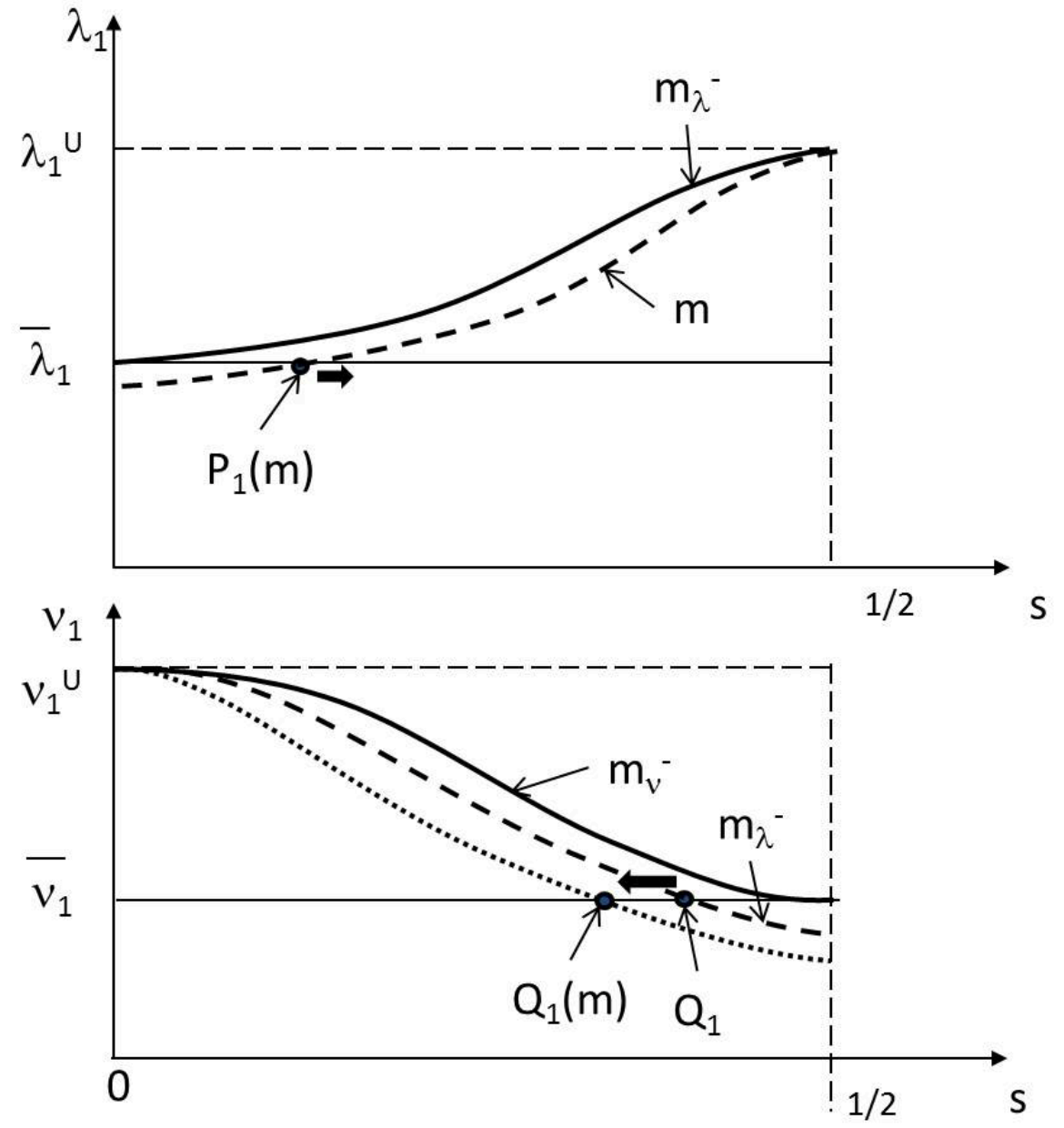

Figure 9: Proof of Theorem 2.2: second case. 